\title{
Vector Differential Calculus
}

\section{Eckhard MS HITZER*}

(Received Feb. 22, 2002)

\begin{abstract}
This paper treats the fundamentals of the vector differential calculus part of universal geometric calculus. Geometric calculus simplifies and unifies the structure and notation of mathematics for all of science and engineering, and for technological applications. In order to make the treatment self-contained, I first compile all important geometric algebra relationships, which are necessary for vector differential calculus. Then differentiation by vectors is introduced and a host of major vector differential and vector derivative relationships is proven explicitly in a very elementary step by step approach. The paper is thus intended to serve as reference material, giving details, which are usually skipped in more advanced discussions of the subject matter.
\end{abstract}

Key Words : Geometric Calculus, Geometric Algebra, Clifford Algebra, Vector Derivative, Vector Differential Calculus

\section{Introduction}

"Now faith is being sure of what we hope for and certain of what we do not see. This is what the ancients were commended for. By faith we understand that the universe was formed at God's command, so that what is seen was not made out of what was visible." [7]

The German $19^{\text {th }}$ century mathematician H. Grassmann had the clear vision, that his "extension theory (now developed to geometric calculus) ... forms the keystone of the entire structure of mathematics."[6] The algebraic "grammar" of this universal form of calculus is geometric algebra (or Clifford algebra). That geometric calculus is a truly unifying approach to all of calculus will be demonstrated here by developing the vector differential calculus part of geometric calculus.

The basic geometric algebra necessary for this is compiled in section 2. Then section 3 develops vector differential calculus with the help of few simple definitions. This approach is generically coordinate free, and fully shows both the concrete and abstract geometric and algebraic beauty of the "keystone" of mathematics.

The underlying strategy of this paper is to demonstrate the proofs for all common formulas of vector differential calculus

\footnotetext{
* Dept. of Mechanical Engineering
}

in an elementary step by step fashion. Thus enabling the interested reader to ultimately use this article as reference material, where other texts (e.g. [1],[2]) tend both to skip "elementary steps", and to presume, that the reader would be smart enough to fill in the gaps himself. I put the emphasis therefore on thorough proofs and not on comments, interpretations or application.

\section{Basic Geometric Algebra}

This section is a basic summary of important relationships in geometric algebra. For brevity they are stated without proof. This summary mainly serves as a reference section for the vector differential calculus to be developed in the following section. Most of the relationships listed here are to be found in the synopsis of geometric algebra and in chapters 1 and 2 of [1], as well as in chapter 1 of [2], together with relevant proofs. Beyond that [1] and [2] follow a much more didactic approach for newcomers to geometric algebra.

$G(I)$ is the full geometric algebra over all vectors in the n-dimensional unit pseudoscalar $I=\vec{e}_{1} \wedge \vec{e}_{2} \wedge \ldots \wedge \vec{e}_{n}$. $A_{n} \equiv G^{1}(I)$ is the n-dimensional vector sub-space of grade-1 elements in $G(I)$ spanned by $\vec{e}_{1}, \vec{e}_{2}, \ldots, \vec{e}_{n}$. For 
vectors $\vec{a}, \vec{b}, \vec{c} \in A_{n} \equiv G^{1}(I)$ and scalars $\alpha, \beta, \lambda, \tau$;

$G(I)$ has the fundamental properties of

- associativity

$\vec{a}(\vec{b} \vec{c})=(\vec{a} \vec{b}) \vec{c}, \vec{a}+(\vec{b}+\vec{c})=(\vec{a}+\vec{b})+\vec{c}$,

- commutativity

$\alpha \vec{a}=\vec{a} \alpha, \quad \vec{a}+\vec{b}=\vec{b}+\vec{a}$,

- distributivity

$\vec{a}(\vec{b}+\vec{c})=\vec{a} \vec{b}+\vec{a} \vec{c}, \quad(\vec{b}+\vec{c}) \vec{a}=\vec{b} \vec{a}+\vec{c} \vec{a}$,

- linearity

$\alpha(\vec{a}+\vec{b})=\alpha \vec{a}+\alpha \vec{b}=(\vec{a}+\vec{b}) \alpha$,

- scalar square (vector length $|\vec{a}|$ )

$\vec{a}^{2}=\vec{a} \vec{a}=\vec{a} \cdot \vec{a}=|\vec{a}|^{2}$.

The geometric product $\vec{a} \vec{b}$ is related to the (scalar) inner product $\vec{a} \cdot \vec{b}$ and to the (bivector or 2-vector) outer product $\vec{a} \wedge \vec{b}$ by

$$
\vec{a} \vec{b}=\vec{a} \cdot \vec{b}+\vec{a} \wedge \vec{b}
$$

with

$$
\begin{aligned}
& \vec{a} \cdot \vec{b}=\frac{1}{2}(\vec{a} \vec{b}+\vec{b} \vec{a})=\vec{b} \cdot \vec{a}=\vec{a} \vec{b}-\vec{a} \wedge \vec{b}=\langle\vec{a} \vec{b}\rangle_{0}, \\
& \vec{a} \wedge \vec{b}=\frac{1}{2}(\vec{a} \vec{b}-\vec{b} \vec{a})=-\vec{b} \wedge \vec{a}=\vec{a} \vec{b}-\vec{a} \cdot \vec{b}=\langle\vec{a} \vec{b}\rangle_{2}
\end{aligned}
$$

The inner and the outer product are both linear and distributive

$$
\begin{gathered}
\vec{a} \cdot(\alpha \vec{b}+\beta \vec{c})=\alpha \vec{a} \cdot \vec{b}+\beta \vec{a} \cdot \vec{c}, \\
\vec{a} \wedge(\alpha \vec{b}+\beta \vec{c})=\alpha \vec{a} \wedge \vec{b}+\beta \vec{a} \wedge \vec{c} .
\end{gathered}
$$

A unit vector $\hat{a}$ in the direction of $\vec{a}$ is

$$
\hat{a} \equiv \frac{\vec{a}}{|\vec{a}|}, \text { with } \hat{a}^{2}=\hat{a} \hat{a}=1, \vec{a}=\hat{a}|\vec{a}| .
$$

The inverse of a vector is

$$
\vec{a}^{-1}=\frac{1}{\vec{a}} \equiv \frac{\vec{a}}{\vec{a}^{2}}=\frac{\vec{a}}{|\vec{a}|^{2}}=\frac{\hat{a}}{|\vec{a}|} .
$$

A multivector $A$ can be uniquely decomposed into its homogeneous grade $\mathrm{k}$ parts $\left(\langle\rangle_{k}\right.$ grade $k$ selector):

$$
A=\underbrace{\langle A\rangle_{0}}_{\text {scalar }}+\underbrace{\langle A\rangle_{1}}_{\text {vector }}+\underbrace{\langle A\rangle_{2}}_{\text {bivector }}+\ldots+\underbrace{\langle A\rangle_{k}}_{k \text {-vector }}+\ldots+\underbrace{\langle A\rangle_{n}}_{\substack{\text { pseudo } \\ \text { scalar }}}(13)
$$

If $A$ is homogeneous of grade $k$ one often simply writes

$$
A=\langle A\rangle_{k}=A_{k} .
$$

Grade selection is invariant under scalar multiplication

$$
\lambda\langle A\rangle_{k}=\langle\lambda A\rangle_{k} .
$$

The consistent definition of inner and outer products of vectors $\vec{a}$ and $r$-vectors $A_{r}$ is

$$
\begin{aligned}
& \vec{a} \cdot A_{r} \equiv\left\langle\vec{a} A_{r}\right\rangle_{r-1}=\frac{1}{2}\left(\vec{a} A_{r}-(-1)^{r} A_{r} \vec{a}\right), \\
& \vec{a} \wedge A_{r} \equiv\left\langle\vec{a} A_{r}\right\rangle_{r+1}=\frac{1}{2}\left(\vec{a} A_{r}+(-1)^{r} A_{r} \vec{a}\right)
\end{aligned}
$$

By linearity the full geometric product of a vector and a multivector $A$ is then

$$
\vec{a} A=\vec{a} \cdot A+\vec{a} \wedge A .
$$

This extends to the distributive multiplication with arbitrary multivectors $A, B$

$$
\vec{a}(A+B)=\vec{a} A+\vec{a} B .
$$

The inner and outer products of homogeneous multivectors $A_{r}$ and $B_{s}$ are defined ([2], p. 6, (1.21), (1.22)) as

$$
\begin{gathered}
A_{r} \cdot B_{s} \equiv\left\langle A_{r} B_{s}\right\rangle_{|r-s|} \text { for } r, s>0, \\
A_{r} \cdot B_{s} \equiv 0 \text { for } r=0 \text { or } s=0, \\
A_{r} \wedge B_{s} \equiv\left\langle A_{r} B_{s}\right\rangle_{r+s},
\end{gathered}
$$

$$
A_{r} \wedge \lambda=\lambda \wedge A_{r}=\lambda A_{r} \text { for scalar } \lambda \text {. }
$$

The inner (and outer) product is again linear and distributive

$\left(\lambda A_{r}\right) \cdot B_{s}=A_{r} \cdot\left(\lambda B_{s}\right)=\lambda\left(A_{r} \cdot B_{s}\right)=\lambda A_{r} \cdot B_{s},(24)$

$$
\begin{aligned}
& A_{r} \cdot\left(B_{s}+C_{t}\right)=A_{r} \cdot B_{s}+A_{r} \cdot C_{t}, \\
& \lambda\left(B_{s}+C_{t}\right)=\lambda B_{s}+\lambda C_{t} .
\end{aligned}
$$

The reverse of a multivector is

$$
\tilde{A}=\sum_{k=1}^{n}(-1)^{k(k-1) / 2}\langle A\rangle_{k} .
$$

[2] uses a dagger instead of the tilde.

Special examples are

$$
\tilde{\lambda}=\lambda, \tilde{\vec{a}}=\vec{a},(\vec{a} \wedge \vec{b})=\vec{b} \wedge \vec{a}=-\vec{a} \wedge \vec{b},
$$


The scalar magnitude $|A|$ of a multivector $A$ is

$$
|A|^{2} \equiv \underbrace{\tilde{A} * A}_{\text {scalar product }} \equiv\langle A\rangle_{0}{ }^{2}+\sum_{r=1}^{n}\langle\tilde{A}\rangle_{r} \cdot\langle A\rangle_{r},
$$

where the separate term $\langle A\rangle_{0}^{2}$ is in particular due to the definition of the inner product in [2], p. 6, (1.21). The magnitude allows to define the inverse for simple $k$-blade vectors

$$
A^{-1} \equiv \frac{\tilde{A}}{|A|^{2}}, \text { with } A^{-1} A=A A^{-1}=1 .
$$

Alternative ways to express $\vec{a} \in A_{n} \equiv G^{1}(I)$ are

$$
I \wedge \vec{a}=0 \text { or } I \vec{a}=I \cdot \vec{a} \text {. }
$$

The projection of $\vec{a}$ into $A_{n} \equiv G^{1}(I)$ is

$$
P_{I}(\vec{a})=P(\vec{a}) \equiv \sum_{k=1}^{n} \vec{a}^{k} \vec{a}_{k} \cdot \vec{a}=\sum_{k=1}^{n} \vec{a}_{k} \vec{a}^{k} \cdot \vec{a},
$$

where $\vec{a}^{k}$ is the reciprocal frame defined by

$$
\vec{a}^{k} \cdot \vec{a}_{j}=\delta_{j}^{k}=\text { Kronecker delta }=\left\{\begin{array}{l}
1 \text { if } j=k \\
0 \text { if } j \neq k
\end{array} .\right.
$$

A general convention is that inner products $\vec{a} \cdot \vec{b}$ and outer products $\vec{a} \wedge \vec{b}$ have priority over geometric products $\vec{a} \vec{b}$, e.g.

$$
\vec{a} \cdot \vec{b} \vec{c} \wedge \vec{d} \vec{e}=(\vec{a} \cdot \vec{b})(\vec{c} \wedge \vec{d}) \vec{e} .
$$

The projection of a multivector $B$ on a subspace described by a simple $m$-vector

(m-blade)

$$
\begin{aligned}
A_{m}=\vec{a}_{1} \wedge \vec{a}_{2} \wedge \ldots \wedge \vec{a}_{m}, m \leq n \text { is } \\
P_{A}(B) \equiv \underbrace{(B \cdot A) \cdot A^{-1}}_{\text {general }}=\underbrace{A^{-1} \cdot\left(A \cdot B_{(s)}\right)}_{\text {degree dependent }}, \\
P_{A}\left(\langle B\rangle_{0}\right) \equiv\langle B\rangle_{0}, \quad P_{A}\left(\langle B\rangle_{n}\right) \equiv\langle B\rangle_{n} \cdot A A^{-1},
\end{aligned}
$$

the exceptions for scalars $\langle B\rangle_{0}$ and pseudoscalars $\langle B\rangle_{n}$ being again due to the definition of the inner product in [2], p. 6, (1.21). A projection of one factor of an inner product has the effect

$$
\vec{a} \cdot P(\vec{b})=P(\vec{a}) \cdot P(\vec{b})=P(\vec{a}) \cdot \vec{b}
$$

For a multivector $B \in G\left(A_{m}\right)$, with $A=A_{m}$ we have

$(\vec{a} \wedge B) \cdot A=(\vec{a} \wedge B) A=\vec{a} \cdot(B A)$ if $\vec{a} \wedge A=0$.

Reordering rules for products of homogeneous multivector are

$$
\begin{gathered}
A_{r} \cdot B_{s}=(-1)^{r(s-r)} B_{s} \cdot A_{r} \text { for } r \leq s, \\
A_{r} \wedge B_{s}=(-1)^{r s} B_{s} \wedge A_{r} .
\end{gathered}
$$

Elementary combinations that occur often are

$\vec{a} \cdot(\vec{b} \wedge \vec{c})=(\vec{a} \cdot \vec{b}) \vec{c}-(\vec{a} \cdot \vec{c}) \vec{b}=\vec{a} \cdot \vec{b} \vec{c}-\vec{a} \cdot \vec{c} \vec{b},(41)$

$(\vec{a} \wedge \vec{b}) \cdot(\vec{c} \wedge \vec{d})=\vec{a} \cdot(\vec{b} \cdot(\vec{c} \wedge \vec{d}))=$

$(\vec{a} \cdot \vec{d})(\vec{b} \cdot \vec{c})-(\vec{a} \cdot \vec{c})(\vec{b} \cdot \vec{d})$,

$(\vec{a} \wedge \vec{b})^{2}=(\vec{a} \wedge \vec{b}) \cdot(\vec{a} \wedge \vec{b})=(\vec{a} \cdot \vec{b})^{2}-\vec{a}^{2} \vec{b}^{2}=$

$-(\vec{b} \wedge \vec{a}) \cdot(\vec{a} \wedge \vec{b})=-|\vec{a} \wedge \vec{b}|^{2}$,

and the Jacobi identity

$$
\vec{a} \cdot(\vec{b} \wedge \vec{c})+\vec{b} \cdot(\vec{c} \wedge \vec{a})+\vec{c} \cdot(\vec{a} \wedge \vec{b})=0 .
$$

The commutator product of multivectors $A, B$ is

$$
A \times B \equiv \frac{1}{2}(A B-B A) .
$$

One useful identity using it is

$$
(\vec{a} \wedge \vec{b}) \times A=\vec{a} \vec{b} \cdot A-A \cdot \vec{a} \vec{b}=\vec{a} \vec{b} \wedge A-A \wedge \vec{a} \vec{b} \text {.(46) }
$$

The commutator product is to be distinguished from the cross product, which is strictly limited to the three-dimensional Euclidean case with unit pseudoscalar $I_{3}$ :

$\vec{a} \times \vec{b} \equiv(\vec{b} \wedge \vec{a}) I_{3}=-(\vec{a} \wedge \vec{b}) I_{3}$.

For more on basic geometric algebra I refer to [1], [2] and to section 3 of [3].

\section{Vector Differential Calculus}

This section shows how to differentiate functions on linear subspaces of the universal geometric algebra $G$ by vectors. It has wide applications particularly to mechanics and physics in general [1]. Separate concepts of gradient, divergence and curl merge into a single concept of vector derivative, united by the geometric product.

The relationship of differential and derivative is clarified. The Taylor expansion (P. 12) is applied to important examples, 
yielding e.g. the Legendre polynomials (P. 36). The adjoint (Def. 57) and the integrability (P. 42, etc.) of multivector functions are defined and discussed. Throughout this section a number of basic differentials and derivations are performed explicitly illustrating ease and power of the calculus.

Since my emphasis here is on explicit step by step proofs, I refer the reader, who is interested in the philosophy, comments and interpretation to the literature ([1]-[5]).

As for the notation: P. 7 refers to proposition 7 of this section. Def. 13 refers to definition 13 of this section. (6) refers to equation number (6) in the previous section on basic geometric algebra.

Standard definitions of continuity and scalar differentiability apply to multivector-valued functions, because the scalar product determines a unique "distance" $|A-B|$ between two elements $A, B \in G(I)$.

\section{Definition 1 (directional derivative)}

$F=F(\vec{x})$ multivector-valued function of a vector variable

$\vec{x}$ defined on an $n$-dimensional vector space $A_{n}=G^{1}(I)$, I unit pseudoscalar. $\vec{a} \in A_{n}$.

$\vec{a} \cdot \vec{\partial} F \equiv \frac{d F(\vec{x}+\vec{a} \tau)}{d \tau}=\lim _{\tau \rightarrow 0} \frac{d F(\vec{x}+\vec{a} \tau)-d F(\vec{x})}{\tau}$

Nomenclature: derivative of $F$ in the direction $\vec{a}$,

$\vec{a}$-derivative of $F$. ([1] uses $\nabla \equiv \vec{\partial}$, [2] uses $\partial \equiv \vec{\partial}$.)

Proposition 2 (distributivity w.r.t. vector argument)

$$
(\vec{a}+\vec{b}) \cdot \vec{\partial} F=\vec{a} \cdot \vec{\partial} F+\vec{b} \cdot \vec{\partial} F, \vec{a}, \vec{b} \in A_{n}
$$

\section{Proof 2}

$$
\begin{aligned}
& (\vec{a}+\vec{b}) \cdot \vec{\partial} F=\lim _{\tau \rightarrow 0} \frac{F(\vec{x}+\vec{b}+\tau \vec{a})-F(\vec{x})}{\tau}= \\
& \lim _{\tau \rightarrow 0}\left\{\frac{F(\vec{x}+\tau \vec{b}+\tau \vec{a})-F(\vec{x}+\tau \vec{b})}{\tau}\right. \\
& \left.+\frac{F(\vec{x}+\tau \vec{b})-F(\vec{x})}{\tau}\right\}
\end{aligned}
$$$$
\stackrel{d e f 1}{=} \lim _{\tau \rightarrow 0} \vec{a} \cdot \vec{\partial} F(\vec{x}+\tau \vec{b})+\vec{b} \cdot \vec{\partial} F=\vec{a} \cdot \vec{\partial} F+\vec{b} \cdot \vec{\partial} F
$$

\section{Proposition 3}

For scalar $\lambda$

$$
(\lambda \vec{a}) \cdot \vec{\partial} F=\lambda(\vec{a} \cdot \vec{\partial} F)
$$

\section{Proof 3}

$$
(\lambda \vec{a}) \cdot \vec{\partial} F=\lim _{\tau \rightarrow 0} \frac{F(\vec{x}+\lambda \vec{a} \tau)-F(\vec{x})}{\tau}
$$

case 1: $\lambda \neq 0$

$$
\begin{aligned}
& (\lambda \vec{a}) \cdot \vec{\partial} F=\lim _{\tau \rightarrow 0} \lambda \frac{F(\vec{x}+\vec{a}(\lambda \tau))-F(\vec{x})}{\lambda \tau} \\
& \underset{r p}{\lambda \neq 0} \lambda \lim _{\tau^{\prime} \rightarrow 0} \frac{F\left(\vec{x}+\vec{a} \tau^{\prime}\right)-F(\vec{x})}{\tau^{\prime}}=\lambda(\vec{a} \cdot \vec{\partial} F)
\end{aligned}
$$

(rp: reparametrization: $\tau \rightarrow \tau^{\prime}=\lambda \tau$ )

case 2: $\lambda=0$

$(\lambda \vec{a}) \cdot \vec{\partial} F \stackrel{\operatorname{def} 1}{=} \lim _{\tau \rightarrow 0} \frac{F(\vec{x})-F(\vec{x})}{\tau}=0=0(\vec{a} \cdot \vec{\partial} F)$.

Proposition 4 (distributivity w.r.t. multivector-valued function)

$$
\vec{a} \cdot \vec{\partial}(F+G)=\vec{a} \cdot \vec{\partial} F+\vec{a} \cdot \vec{\partial} G
$$

$F=F(\vec{x}), G=G(\vec{x})$ multivector-valued functions of a vector variable $\vec{x}$. In the notation of Def. 13:

$$
\underline{F+G}=\underline{F}+\underline{G} .
$$

\section{Proof 4}

$\vec{a} \cdot \vec{\partial}(F+G) \stackrel{\operatorname{def} 1}{=}$

$\lim _{\tau \rightarrow 0} \frac{F(\vec{x}+\vec{a} \tau)+G(\vec{x}+\vec{a} \tau)-F(\vec{x})-G(\vec{x})}{\tau}=$

$\lim _{\tau \rightarrow 0} \frac{F(\vec{x}+\vec{a} \tau)-F(\vec{x})}{\tau}+$

$\lim _{\tau \rightarrow 0} \frac{G(\vec{x}+\vec{a} \tau)-G(\vec{x})}{\tau} \stackrel{\operatorname{def} 1}{=} \vec{a} \cdot \vec{\partial} F+\vec{a} \cdot \vec{\partial} G$.

Proposition 5 (product rule)

$$
\vec{a} \cdot \vec{\partial}(F G)=(\vec{a} \cdot \vec{\partial} F) G+F(\vec{a} \cdot \vec{\partial} G)
$$

In the notation of Def. 13:

$$
\underline{F G}=\underline{F G}+F \underline{G} .
$$

\section{Proof 5}

$$
\begin{aligned}
& \vec{a} \cdot \vec{\partial}(F G) \stackrel{\text { def } 1}{=} \\
& \lim _{\tau \rightarrow 0} \frac{F(\vec{x}+\vec{a} \tau) G(\vec{x}+\vec{a} \tau)-F(\vec{x}) G(\vec{x})}{\tau} \\
& =\lim _{\tau \rightarrow 0}\left\{\frac{F(\vec{x}+\vec{a} \tau) G(\vec{x}+\vec{a} \tau)-F(\vec{x}) G(\vec{x}+\vec{a} \tau)+}{\tau}\right.
\end{aligned}
$$




$$
\begin{aligned}
& +F(\vec{x}) G(\vec{x}+\vec{a} \tau)-F(\vec{x}) G(\vec{x}) \\
& =\lim _{\tau \rightarrow 0}\left\{\frac{F(\vec{x}+\vec{a} \tau)-F(\vec{x})}{\tau} G(\vec{x}+\vec{a} \tau)\right. \\
& \left.+F(\vec{x}) \frac{G(\vec{x}+\vec{a} \tau)-G(\vec{x})}{\tau}\right\} \\
& \quad \operatorname{def} 1 \\
& =(\vec{a} \cdot \vec{\partial} F) \lim _{\tau \rightarrow 0} G(\vec{x}+\vec{a} \tau)+F(\vec{a} \cdot \vec{\partial} G) \\
& =(\vec{a} \cdot \vec{\partial} F) G+F(\vec{a} \cdot \vec{\partial} G)
\end{aligned}
$$

Proposition 6 (grade invariance)

$$
\vec{a} \cdot \vec{\partial}\langle F\rangle_{k}=\langle\vec{a} \cdot \vec{\partial} F\rangle_{k}
$$

$\vec{a} \cdot \vec{\partial}$ is therefore said to be a scalar differential operator.

\section{Proof 6}

$$
\begin{aligned}
& \vec{a} \cdot \vec{\partial}\langle F\rangle_{k}{ }_{\text {def } 1}^{=} \lim _{\tau \rightarrow 0} \frac{\langle F(\vec{x}+\vec{a} \tau)\rangle_{k}-\langle F(\vec{x})\rangle_{k}}{\tau} \\
& \stackrel{\tau=\text { scalar }}{=} \lim _{\tau \rightarrow 0}\left\langle\frac{F(\vec{x}+\vec{a} \tau)-F(\vec{x})}{\tau}\right\rangle_{k}
\end{aligned}
$$

$\stackrel{\operatorname{def} 1}{=}\langle\vec{a} \cdot \vec{\partial} F\rangle_{k}$.

Proposition 7 (scalar chain rule)

$$
\vec{a} \cdot \vec{\partial} F=(\vec{a} \cdot \vec{\partial} \lambda) \frac{d F}{d \lambda}
$$

$F=F(\lambda(\vec{x})), \quad \lambda=\lambda(\vec{x})$ scalar valued function.

\section{Proof 7}

Using the Taylor expansions:

$F(\lambda+\tau \Delta \lambda)=F(\lambda)+\tau \Delta \lambda \frac{d F}{d \lambda}+\frac{\tau^{2}(\Delta \lambda)^{2}}{2} \frac{d^{2} F}{d \lambda^{2}}+$

$$
\lambda(\vec{x}+\tau \vec{a}) \stackrel{P 12}{=} \lambda(\vec{x})+\tau \vec{a} \cdot \vec{\partial} \lambda(\vec{x})+\frac{\tau}{2}(\vec{a} \cdot \vec{\partial})^{2} \lambda(\vec{x})+\ldots
$$

we have

$$
\begin{aligned}
& \vec{a} \cdot \vec{\partial} F(\lambda(\vec{x}))=\lim _{\tau \rightarrow 0} \frac{F(\lambda(\vec{x}+\tau \vec{a}))-F(\lambda(\vec{x}))}{\tau}= \\
& \lim _{\tau \rightarrow 0} \frac{F(\lambda(\vec{x})+\tau \vec{a} \cdot \vec{\partial} \lambda(\vec{x}))-F(\lambda(\vec{x}))}{\tau} \stackrel{\text { Taylor }}{=} \\
& \lim _{\tau \rightarrow 0} \frac{F(\lambda(\vec{x}))+\tau\{\vec{a} \cdot \vec{\partial} \lambda(\vec{x})\} \frac{d F}{d \lambda}-F(\lambda(\vec{x}))}{\tau}=
\end{aligned}
$$

$=(\vec{a} \cdot \vec{\partial} \lambda) \frac{d F}{d \lambda}$.

Proposition 8 (identity)

$$
\vec{a} \cdot \vec{\partial} \vec{x}=\vec{a} .
$$

\section{Proof 8}

$F(\vec{x})=\vec{x}, \vec{a} \cdot \vec{\partial} \vec{x}=\lim _{\tau \rightarrow 0} \frac{\vec{x}+\tau \vec{a}-\vec{x}}{\tau}=\lim _{\tau \rightarrow 0} \vec{a}=\vec{a}$.

Proposition 9 (constant function)

$A$ independent of $\vec{x}$ :

$$
\vec{a} \cdot \vec{\partial} A=0
$$

\section{Proof 9}

$F(\vec{x})=A, \vec{a} \cdot \vec{\partial} A \stackrel{\text { def } 1}{=} \lim _{\tau \rightarrow 0} \frac{A-A}{\tau}=0$.

Proposition 10 (vector length)

$$
\vec{a} \cdot \vec{\partial}|\vec{x}|=\frac{\vec{a} \cdot \vec{x}}{|\vec{x}|}=\vec{a} \cdot \hat{x} .
$$

$\hat{x} \equiv \frac{\vec{X}}{|\vec{x}|}$ unit vector in the direction of $\vec{x}$

\section{Proof 10}

$\vec{a} \cdot \overrightarrow{\partial \vec{x}^{2}}=(\vec{a} \cdot \vec{\partial} \vec{x}) \vec{x}+\vec{x}(\vec{a} \cdot \vec{\partial} \vec{x}) \stackrel{P 8}{=} \vec{a} \vec{x}+\vec{x} \vec{a}=2 \vec{a} \cdot \vec{x}$, $\vec{a} \cdot \vec{\partial}|\vec{x}|^{2}=(\vec{a} \cdot \vec{\partial}|\vec{x}|)|\vec{x}|+|\vec{x}|(\vec{a} \cdot \vec{\partial}|\vec{x}|)=2|\vec{x}|(\vec{a} \cdot \vec{\partial}|\vec{x}|)$, $\vec{x}^{2}=|\vec{x}|^{2} \Rightarrow \vec{a} \cdot \vec{\partial} \vec{x}^{2}=\vec{a} \cdot \vec{\partial}|\vec{x}|^{2}$ $\Rightarrow \vec{a} \cdot \vec{x}=|\vec{x}|(\vec{a} \cdot \vec{\partial}|\vec{x}|) \Rightarrow \vec{a} \cdot \vec{\partial}|\vec{x}|=\frac{\vec{a} \cdot \vec{x}}{|\vec{x}|}=\vec{a} \cdot \hat{x}$.

Proposition 11 (direction function)

$$
\vec{a} \cdot \vec{\partial} \hat{x}=\frac{\vec{a}-\vec{a} \cdot \hat{x} \hat{x}}{|\vec{x}|}=\frac{\hat{x} \hat{x} \wedge \vec{a}}{|\vec{x}|} .
$$

\section{Proof 11}

$\vec{a} \cdot \vec{\partial} \hat{x}=\vec{a} \cdot \vec{\partial} \frac{\vec{x}}{|\vec{x}|}=\frac{P 5 \cdot \vec{\partial} \vec{x}}{|\vec{x}|}+\vec{x} \vec{a} \cdot \vec{\partial} \frac{1}{|\vec{x}|} \stackrel{P 7,8}{=}$ $\frac{\vec{a}}{|\vec{x}|}+\vec{x}(\vec{a} \cdot \vec{\partial} \mid \vec{x}) \frac{d \frac{1}{|\vec{x}|}}{d|\vec{x}|}=\frac{P 10}{|\vec{x}|}+\vec{x}(\vec{a} \cdot \hat{x}) \frac{-1}{|\vec{x}|^{2}}=$
$\frac{\vec{a}}{|\vec{x}|}-\hat{x}(\vec{a} \cdot \hat{x}) \frac{1}{|\vec{x}|}=\frac{\vec{a}-\vec{a} \cdot \hat{x} \hat{x}}{|\vec{x}|}=\frac{\hat{x} \hat{x} \vec{a}-\hat{x} \hat{x} \cdot \vec{a}}{|\vec{x}|}=$ 
$=\frac{\hat{x}(\hat{x} \cdot \vec{a}+\hat{x} \wedge \vec{a})-\hat{x} \hat{x} \cdot \vec{a}}{|\vec{x}|}=\frac{\hat{x} \hat{x} \wedge \vec{a}}{|\vec{x}|}$.

Proposition 12 (Taylor expansion)

$F(\vec{x}+\vec{a})=\exp (\vec{a} \cdot \vec{\partial}) F(\vec{x})=\sum_{k=0}^{\infty} \frac{(\vec{a} \cdot \vec{\partial})^{k}}{k !} F(\vec{x})$.

\section{Proof 12}

This proof is done without referring to P7 to P11!

$G(\tau) \equiv F(\vec{x}+\vec{a} \tau)$

$\Rightarrow \frac{d G(0)}{d \tau}=\left.\frac{d F(\vec{x}+\vec{a} \tau)}{d \tau}\right|_{\tau=0} \stackrel{\operatorname{def} 1}{=} \vec{a} \cdot \vec{\partial} F$

$\Rightarrow \frac{d^{2} G(0)}{d \tau^{2}}=\left.\frac{d}{d \tau} \frac{d F(\vec{x}+\vec{a} \tau)}{d \tau}\right|_{\tau=0} \stackrel{\text { def } 1}{=}$

$\left.\vec{a} \cdot \vec{\partial} \frac{d F(\vec{x}+\vec{a} \tau)}{d \tau}\right|_{\tau=0} \stackrel{\operatorname{def} 1}{=} \vec{a} \cdot \vec{\partial}(\vec{a} \cdot \vec{\partial} F(\vec{x}))=$

$(\vec{a} \cdot \vec{\partial})^{2} F(\vec{x})$.

General: $\frac{d^{k} G(0)}{d \tau^{k}}=(\vec{a} \cdot \vec{\partial})^{k} F(\vec{x})$.

The Taylor series for $G$ is:

$G(1)=G(0+1)=G(0)+\frac{d G(0)}{d \tau}+\frac{1}{2} \frac{d^{2} G(0)}{d \tau^{2}}+\ldots$

$=\sum_{k=0}^{\infty} \frac{1}{k !} \frac{d^{k} G(0)}{d \tau^{k}}$

$\Rightarrow G(1)=F(\vec{x}+\vec{a})=$

$\sum_{k=0}^{\infty} \frac{1}{k !}(\vec{a} \cdot \vec{\partial})^{k} F(\vec{x})=\exp (\vec{a} \cdot \vec{\partial}) F(\vec{x})$.

Definition 13 (continuously differentiable, differential)

$F$ is continuously differentiable at $\vec{x}$ if for each fixed $\vec{a}$ $\vec{a} \cdot \vec{\partial} F(\vec{y})$ exists and is a continuous function of $\vec{y}$ for each $\vec{y}$ in a neighborhood of $\vec{x}$.

If $F$ is defined and continuously differentiable at $\vec{X}$, then, for

fixed $\vec{x}, \vec{a} \cdot \vec{\partial} F(\vec{x})$ is a linear function of $\vec{a}$, the (first) differential of $F$.

$$
\underline{F}(\vec{a}, \vec{x})=F_{\vec{a}}(\vec{x}) \equiv \vec{a} \cdot \vec{\partial} F(\vec{x}) .
$$

([1], p. 107 uses $F^{\prime} \equiv \underline{F}$.)
Suppressing $\vec{x}$, or for fixed $\vec{x}$ :

$$
\underline{F}=\underline{F}(\vec{a})=F_{\vec{a}} \equiv \vec{a} \cdot \vec{\partial} F .
$$

\section{Proposition 14 (linearity)}

$$
\begin{aligned}
& \underline{F}(\vec{a}+\vec{b})=\underline{F}(\vec{a})+\underline{F}(\vec{b}) \\
& \lambda \text { scalar: } \underline{F}(\lambda \vec{a})=\lambda \underline{F}(\vec{a})
\end{aligned}
$$

\section{Proof 14}

Propositions 2 and 3.

Proposition 15 (linear approximation)

For $|\vec{r}|=\left|\vec{x}-\vec{x}_{0}\right|$ sufficiently small:

$$
F(\vec{x})-F\left(\vec{x}_{0}\right) \approx \underline{F}\left(\vec{x}-\vec{x}_{0}\right)=\underline{F}(\vec{x})-\underline{F}\left(\vec{x}_{0}\right) .
$$

\section{Proof 15}

$$
\begin{aligned}
& F(\vec{x})=F(\vec{x}+\vec{r})= \\
& F\left(\vec{x}_{0}\right)+\vec{r} \cdot \vec{\partial} F\left(\vec{x}_{0}\right)+\frac{1}{2}(\vec{r} \cdot \vec{\partial})^{2} F\left(\vec{x}_{0}\right)+\ldots= \\
& F\left(\vec{x}_{0}\right)+|\vec{r}| \hat{r} \cdot \vec{\partial} F\left(\vec{x}_{0}\right)+\frac{|\vec{r}|^{2}}{2}(\hat{r} \cdot \vec{\partial})^{2} F\left(\vec{x}_{0}\right)+\ldots \\
& +\frac{|\vec{r}|^{k}}{2}(\hat{r} \cdot \vec{\partial})^{k} F\left(\vec{x}_{0}\right)+\ldots, \\
& \text { with } \hat{r} \equiv \frac{\vec{r}}{|\vec{r}|} \cdot \text { For sufficiently small }|\vec{r}|: \\
& F(\vec{x})=F\left(\vec{x}_{0}\right)+\vec{r} \cdot \vec{\partial} F\left(\vec{x}_{0}\right)= \\
& F\left(\vec{x}_{0}\right)+\left(\vec{x}-\vec{x}_{0}\right) \cdot \vec{\partial} F\left(\vec{x}_{0}\right)= \\
& F\left(\vec{x}_{0}\right)+\underline{F}\left(\vec{x}-\vec{x}_{0}, \vec{x}_{0}\right)=F\left(\vec{x}_{0}\right)+\underline{F}\left(\vec{x}-\vec{x}_{0}\right)= \\
& F\left(\vec{x}_{0}\right)+\underline{F}(\vec{x})-\underline{F}\left(\vec{x}_{0}\right) \\
& \Rightarrow F(\vec{x})-F\left(\vec{x}_{0}\right) \approx \underline{F}\left(\vec{x}-\vec{x}_{0}\right)=\underline{F}(\vec{x})-\underline{F}\left(\vec{x}_{0}\right) .
\end{aligned}
$$

Proposition 16 (chain rule)

$$
\frac{d F}{d t}(\vec{x}(t))=\left.\left(\frac{d}{d t} \vec{x}(t)\right) \cdot \vec{\partial} F(\vec{x})\right|_{\vec{x}=\vec{x}(t)}
$$

\section{Proof 16}

Using the Taylor expansion

$$
\begin{gathered}
\vec{x}(t+\tau)=\vec{x}(t)+\tau \frac{d}{d t} \vec{x}(t)+\frac{\tau^{2}}{2} \frac{d^{2}}{d t^{2}} \vec{x}(t)+\ldots, \\
\frac{d F}{d t}(\vec{x}(t))=\lim _{\tau \rightarrow 0} \frac{F(\vec{x}(t+\tau))-F(\vec{x}(t))}{\tau} \stackrel{\text { Taylor }}{=} \\
\lim _{\tau \rightarrow 0} \frac{F\left(\vec{x}(t)+\tau \frac{d}{d t} \vec{x}(t)\right)-F(\vec{x}(t))_{\text {def } 1}}{\tau}=
\end{gathered}
$$




$$
=\left.\left(\frac{d}{d t} \vec{x}(t)\right) \cdot \vec{\partial} F(\vec{x})\right|_{\vec{x}=\vec{x}(t)} .
$$

Definition 17 (vector derivative)

Differentiation of $F$ by its argument $\vec{x}$

$\vec{\partial}_{\vec{x}} F(\vec{x})=\vec{\partial} F$,

with the differential operator $\vec{\partial}_{\vec{x}}$, assumed to

(i) have the algebraic properties of a vector in $A_{n} \equiv G^{1}(I)$, I unit pseudoscalar; and

(ii) that $\vec{a} \cdot \vec{\partial}_{\vec{x}}$ with $\vec{a} \in A_{n}$ is $\vec{a} \cdot \vec{\partial}_{\vec{x}} F$ as in Def. 1.

Proposition 18 (algebraic properties of $\vec{\partial}_{\vec{x}}$ )

$$
\begin{gathered}
I \wedge \vec{\partial}_{\vec{x}}{ }^{(31)}=0 \\
I \vec{\partial}_{\vec{x}}{ }^{(31)}=I \cdot \vec{\partial}_{\vec{x}} \\
\vec{\partial}_{\vec{x}}=P_{I}\left(\vec{\partial}_{\vec{x}}\right) \stackrel{(32)}{=} \sum_{k=1}^{n} \vec{a}^{k} \vec{a}_{k} \cdot \vec{\partial}_{\vec{x}},
\end{gathered}
$$

where the $\vec{a}^{k}$ express the algebraic vector properties and the $\vec{a}_{k} \cdot \vec{\partial}_{\vec{x}}$ the scalar differential properties.

\section{Definition 19 (gradient)}

The vector field $\vec{f}=\vec{f}(\vec{x}) \equiv \vec{\partial}_{\vec{x}} \Phi(\vec{x})=\vec{\partial} \Phi$ for a scalar function $\Phi=\Phi(\vec{x})$ is called the gradient of $\Phi$.

\section{Propostion 20 (3-dimensional cross product)}

For $\vec{b}$ independent of $\vec{x} \in A_{3} \equiv G^{1}\left(I_{3}\right)$ :

$$
\vec{a} \cdot \vec{\partial}(\vec{x} \times \vec{b})=\vec{a} \times \vec{b} .
$$

Only here $\times$ means the 3 -dimensional cross product (47), not the commutator product in P. 81.

Proof 20

$$
\begin{aligned}
& \vec{a} \cdot \vec{\partial}(\vec{x} \times \vec{b}) \stackrel{(47)}{=}-\vec{a} \cdot \vec{\partial}\left((\vec{x} \wedge \vec{b}) I_{3}\right)^{P 5}= \\
& {[-\vec{a} \cdot \vec{\partial}(\vec{x} \wedge \vec{b})] I_{3}-(\vec{x} \wedge \vec{b}) \vec{a} \cdot \vec{\partial} I_{3} \underset{P 9}{\stackrel{I_{3}=\text { const }}{=}}} \\
& {[-\vec{a} \cdot \vec{\partial}(\vec{x} \wedge \vec{b})] I_{3}{ }^{(8)}=\left[-\vec{a} \cdot \vec{\partial}\langle\vec{x} \vec{b}\rangle_{2}\right]_{3}{ }_{3}^{P 6}=} \\
& -\langle\vec{a} \cdot \vec{\partial} \vec{x} \vec{b}\rangle_{2} I_{3}{ }^{P 5}=-\langle(\vec{a} \cdot \vec{\partial} \vec{x}) \vec{b}\rangle_{2} I_{3}-\langle\vec{x}(\vec{a} \cdot \vec{\partial} \vec{b})\rangle_{2} I_{3}
\end{aligned}
$$

$$
\stackrel{P 8,9}{=}-\langle\vec{a} \vec{b}\rangle_{2} I_{3} \stackrel{(8)}{=}-(\vec{a} \wedge \vec{b}) I_{3} \stackrel{(47)}{=} \vec{a} \times \vec{b} .
$$

\section{Proposition 21}

$$
\vec{a} \cdot \vec{\partial}\left(\vec{x} \cdot\langle A\rangle_{r}\right)=\vec{a} \cdot\langle A\rangle_{r},
$$

$A$ independent of $\vec{x}$.

\section{Proof 21}

$\vec{a} \cdot \vec{\partial}\left(\vec{x} \cdot\langle A\rangle_{r}\right)^{(16)}=\vec{a} \cdot \vec{\partial}\left\langle\vec{x}\langle A\rangle_{r}\right\rangle_{r-1} \stackrel{P 6}{=}$

$\left\langle\vec{a} \cdot \vec{\partial}\left(\vec{x}\langle A\rangle_{r}\right)\right\rangle_{r-1}{ }^{P 5}=\left\langle(\vec{a} \cdot \vec{\partial} \vec{x})\langle A\rangle_{r}+\vec{x} \vec{a} \cdot \vec{\partial}\langle A\rangle_{r}\right\rangle_{r-1}{ }^{P 8,9}=$ $\left\langle\vec{a}\langle A\rangle_{r}\right\rangle_{r-1} \stackrel{(16)}{=} \vec{a} \cdot\langle A\rangle_{r}$.

\section{Proposition 22}

$$
\vec{a} \cdot \vec{\partial}[\vec{x} \cdot(\vec{x} \wedge \vec{b})]=\vec{a} \cdot(\vec{x} \wedge \vec{b})+\vec{x} \cdot(\vec{a} \wedge \vec{b})
$$

\section{Proof 22}

$\vec{a} \cdot \vec{\partial}[\vec{x} \cdot(\vec{x} \wedge \vec{b})]^{(41)}=\vec{a} \cdot \vec{\partial}\left[\vec{x}^{2} \vec{b}+\vec{x} \cdot \vec{b} \vec{x}\right]^{P 4,5}=$ $\left(\vec{a} \cdot \vec{\partial}^{2}\right) \vec{b}+\vec{x}^{2} \vec{a} \cdot \vec{\partial} \vec{b}+\vec{a} \cdot \vec{\partial}(\vec{x} \cdot \vec{b}) \vec{x}+\vec{x} \cdot \vec{b}(\vec{a} \cdot \vec{\partial} \vec{x})$ $\underset{P 9,21,8}{\stackrel{P}{=}} \vec{a} \cdot \vec{x} \vec{b}-\vec{a} \cdot \vec{b} \vec{x}+\vec{x} \cdot \vec{a} \vec{b}+\vec{x} \cdot \vec{b} \vec{a} \stackrel{(41)}{=}$ $\vec{a} \cdot(\vec{x} \wedge \vec{b})+\vec{x} \cdot(\vec{a} \wedge \vec{b})$.

\section{Proposition 23}

For $\vec{x}^{\prime}$ independent of $\vec{x}$ and $r \equiv|\vec{r}|=\left|\vec{x}-\vec{x}^{\prime}\right|$ :

$$
\vec{a} \cdot \vec{\partial} r=\vec{a} \cdot \frac{\vec{r}}{r}=\vec{a} \cdot \hat{r}
$$

where $\hat{r}=\frac{\vec{r}}{r}$.

\section{Proof 23}

Compare [1], p. 681. $r^{2}=\left(\vec{x}-\vec{x}^{\prime}\right)\left(\vec{x}-\vec{x}^{\prime}\right)$, then

$\vec{a} \cdot \vec{\partial} r^{2} \stackrel{P 5}{=}$

$\left[\vec{a} \cdot \vec{\partial}\left(\vec{x}-\vec{x}^{\prime}\right)\right]\left(\vec{x}-\vec{x}^{\prime}\right)+\left(\vec{x}-\vec{x}^{\prime}\right)\left[\vec{a} \cdot \vec{\partial}\left(\vec{x}-\vec{x}^{\prime}\right)\right]$

$\stackrel{P 4,9}{=} \vec{a}\left(\vec{X}-\vec{x}^{\prime}\right)+\left(\vec{x}-\vec{x}^{\prime}\right) \vec{a}=2 \vec{a} \cdot \vec{r}$,

$\vec{a} \cdot \vec{\partial} r^{2} \stackrel{P 5}{=} 2 r(\vec{a} \cdot \vec{\partial} r) \Rightarrow 2 \vec{a} \cdot \vec{r}=2 r(\vec{a} \cdot \vec{\partial} r)$

$\Rightarrow \vec{a} \cdot \vec{\partial} r=\vec{a} \cdot \frac{\vec{r}}{r}=\vec{a} \cdot \hat{r}$.

\section{Proposition 24}




$$
\vec{a} \cdot \vec{\partial} \hat{r}=\frac{\hat{r} \hat{r} \wedge \vec{a}}{r}
$$

\section{Proof 24}

Compare [1], p. 681.

$\vec{a} \cdot \vec{\partial} \hat{r}=\vec{a} \cdot \frac{\vec{r}}{r}=\frac{P 5}{r} \vec{a} \cdot \vec{\partial} \vec{r}+\vec{r} \vec{a} \cdot \vec{\partial} \frac{1}{r}=$

$\frac{1}{r} \vec{a} \cdot \vec{\partial}\left(\vec{x}-\vec{x}^{\prime}\right)-\vec{r} \frac{1}{r^{2}} \vec{a} \cdot \vec{\partial} r \stackrel{P 8,23}{=} \frac{1}{r} \vec{a}-\frac{\hat{r}}{r} \vec{a} \cdot \hat{r}=$ $\frac{\hat{r} \hat{r} \vec{a}-\hat{r}(\hat{r} \cdot \vec{a})^{(8)}}{r}=\frac{\hat{r} \hat{r} \wedge \vec{a}}{r}$.

\section{Proposition 25}

$$
\vec{a} \cdot \vec{\partial}(\hat{r} \cdot \vec{a})=\frac{|\hat{r} \wedge \vec{a}|^{2}}{r} .
$$

\section{Proof 25}

$\vec{a} \cdot \vec{\partial}(\hat{r} \cdot \vec{a})=\vec{a} \cdot \vec{\partial}\left(\frac{\vec{r} \cdot \vec{a}}{r}\right) \stackrel{P 5}{=}$ $\frac{1}{r} \vec{a} \cdot \vec{\partial}(\vec{r} \cdot \vec{a})+\vec{r} \cdot \vec{a}(\vec{a} \cdot \vec{\partial}) \frac{1}{r}{ }^{P 7,23}=$

$\frac{1}{r} \vec{a} \cdot \vec{\partial}\left(\vec{x} \cdot \vec{a}-\vec{x}^{\prime} \cdot \vec{a}\right)-\frac{\vec{r} \cdot \vec{a}}{r^{2}} \vec{a} \cdot \hat{r}=$

$\frac{1}{r} \vec{a} \cdot \vec{a}-\frac{(\hat{r} \cdot \vec{a})^{2}}{r}=\frac{(11)}{=} \frac{\hat{r}^{2} \vec{a}^{2}-(\hat{r} \cdot \vec{a})^{2}}{r}=$

$\frac{(\hat{r} \wedge \vec{a}) \cdot(\vec{a} \wedge \hat{r})}{r} \stackrel{(43)}{=} \frac{|\hat{r} \wedge \vec{a}|^{2}}{r}$.

Proposition 26

$$
\vec{a} \cdot \vec{\partial}(\hat{r} \wedge \vec{a})=\frac{\hat{r} \cdot \vec{a} \vec{a} \wedge \hat{r}}{r} .
$$

\section{Proof 26}

$\vec{a} \cdot \vec{\partial}(\hat{r} \wedge \vec{a}) \stackrel{(8)}{=} \vec{a} \cdot \vec{\partial}(\hat{r} \vec{a}-\hat{r} \cdot \vec{a}) \stackrel{P 4}{=}$

$\vec{a} \cdot \vec{\partial}(\hat{r} \vec{a})-\vec{a} \cdot \vec{\partial}(\hat{r} \cdot \vec{a}) \stackrel{P 5,25}{=}$

$\vec{a} \cdot \vec{\partial}(\hat{r}) \vec{a}+\hat{r} \vec{a} \cdot \vec{\partial} \vec{a}-\frac{|\hat{r} \wedge \vec{a}|^{2}}{r} \underset{(43)}{=}$

$\frac{\hat{r} \hat{r} \wedge \vec{a}}{r} \vec{a}-\frac{(\vec{a} \wedge \hat{r})(\hat{r} \wedge \vec{a})}{r} \underset{(40)}{=}$

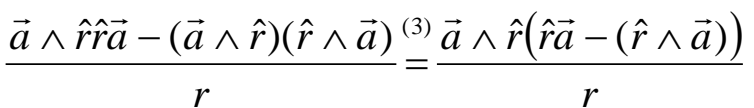

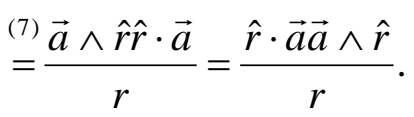

Proposition 27

$$
\vec{a} \cdot \vec{\partial}|\hat{r} \wedge \vec{a}|=-\frac{\hat{r} \cdot \vec{a}|\hat{r} \wedge \vec{a}|}{r} .
$$

$\vec{a} \cdot \vec{\partial}|\hat{r} \wedge \vec{a}|^{2}=2|\hat{r} \wedge \vec{a}| \vec{a} \cdot \vec{\partial}|\hat{r} \wedge \vec{a}|$,

$\vec{a} \cdot \vec{\partial}|\hat{r} \wedge \vec{a}|^{2} \stackrel{(43)}{=} \vec{a} \cdot \vec{\partial}[(\hat{r} \wedge \vec{a}) \cdot(\vec{a} \wedge \hat{r})]=$

$\vec{a} \cdot \vec{\partial}\left[\hat{r}^{2} \vec{a}^{2}-(\hat{r} \cdot \vec{a})^{2}\right]^{P 5} \vec{a} \cdot \vec{\partial} \vec{a}^{2}-2(\hat{r} \cdot \vec{a}) \vec{a} \cdot \vec{\partial}(\hat{r} \cdot \vec{a})$

$\stackrel{P 9,25}{=}-2(\hat{r} \cdot \vec{a}) \frac{|\hat{r} \wedge \vec{a}|^{2}}{r}$

$\Rightarrow 2|\hat{r} \wedge \vec{a}| \vec{a} \cdot \vec{\partial}|\hat{r} \wedge \vec{a}|=-2 \frac{(\hat{r} \cdot \vec{a})|\hat{r} \wedge \vec{a}|^{2}}{r}$

$\Rightarrow \vec{a} \cdot \vec{\partial}|\hat{r} \wedge \vec{a}|=-\frac{(\hat{r} \cdot \vec{a})|\hat{r} \wedge \vec{a}|}{r}$.

\section{Proposition 28}

$$
\vec{a} \cdot \vec{\partial} \frac{1}{\vec{r}}=-\frac{1}{\vec{r}} \vec{a} \frac{1}{\vec{r}} .
$$

\section{Proof 28}

$\vec{a} \cdot \vec{\partial} \frac{1}{\vec{r}} \stackrel{(12)}{=} \vec{a} \cdot \vec{\partial} \frac{\vec{r}}{r^{2}}=\frac{1}{r^{2}} \vec{a} \cdot \vec{\partial} \vec{r}+\vec{r} \vec{a} \cdot \vec{\partial} \frac{1}{r^{2}}=$

$\frac{1}{r^{2}} \vec{a}+\vec{r}\left(-\frac{2}{r^{3}}\right) \vec{a} \cdot \vec{\partial} r \stackrel{P 23}{=} \frac{1}{r^{2}} \vec{a}-2 \frac{\vec{r}}{r^{3}} \vec{a} \cdot \hat{r} \stackrel{(12),(11)}{=}$

$\frac{1}{\vec{r}} \frac{1}{\vec{r}} \vec{a}-2 \frac{1}{\vec{r}} \vec{a} \cdot \frac{1}{\vec{r}}=\frac{(7)}{\vec{r}} \frac{1}{\vec{r}} \vec{a}-\frac{1}{\vec{r}} \vec{a} \frac{1}{\vec{r}}-\frac{1}{\vec{r}} \frac{1}{\vec{r}} \vec{a}=$ $-\frac{1}{\vec{r}} \vec{a} \frac{1}{\vec{r}}$.

\section{Proposition 29}

$$
\vec{a} \cdot \vec{\partial} \frac{1}{r^{2}}=-2 \frac{\vec{a} \cdot \hat{r}}{r^{3}} .
$$

\section{Proof 29}

$\vec{a} \cdot \vec{\partial} \frac{1}{r^{2}}=-\frac{2}{r^{3}} \vec{a} \cdot \vec{\partial} r \stackrel{P 23}{=}-\frac{2}{r^{3}} \vec{a} \cdot \vec{\partial} \hat{r}$.

\section{Proposition 30}

$$
\frac{1}{2}(\vec{a} \cdot \vec{\partial})^{2} \frac{1}{r^{2}}=\frac{3(\vec{a} \cdot \hat{r})^{2}-|\hat{r} \wedge \vec{a}|^{2}}{r^{4}} .
$$

\section{Proof 30}

$\frac{1}{2}(\vec{a} \cdot \vec{\partial})^{2} \frac{1}{r^{2}} \stackrel{P 29}{=} \frac{1}{2} \vec{a} \cdot \vec{\partial}\left(-2 \frac{\vec{a} \cdot \hat{r}}{r^{3}}\right)^{P 5}=$

$-\left(\vec{a} \cdot \vec{\partial} \frac{1}{r^{3}}\right) \vec{a} \cdot \hat{r}-\frac{1}{r^{3}} \vec{a} \cdot \vec{\partial}(\vec{a} \cdot \hat{r}) \stackrel{P 7,23,25}{=}$

$\frac{3 \vec{a} \cdot \hat{r}}{r^{4}} \vec{a} \cdot \hat{r}-\frac{1}{r^{3}} \frac{|\hat{r} \wedge \vec{a}|^{2}}{r}=\frac{3(\vec{a} \cdot \hat{r})^{2}-|\hat{r} \wedge \vec{a}|^{2}}{r^{4}}$. 


\section{Proposition 31}

$\frac{1}{6}(\vec{a} \cdot \vec{\partial})^{3} \frac{1}{r^{2}}=\frac{-4(\vec{a} \cdot \hat{r})^{3}+4|\hat{r} \wedge \vec{a}|^{2} \vec{a} \cdot \hat{r}}{r^{5}}$.

\section{Proof 31}

$\frac{1}{6}(\vec{a} \cdot \vec{\partial})^{3} \frac{1}{r^{2}}=\frac{1}{3}(\vec{a} \cdot \vec{\partial}) \frac{1}{2}(\vec{a} \cdot \vec{\partial})^{2} \frac{1}{r^{2}} \stackrel{P 30}{=}$

$\frac{1}{3}(\vec{a} \cdot \vec{\partial}) \frac{3(\vec{a} \cdot \hat{r})^{2}-|\hat{r} \wedge \vec{a}|^{2}}{r^{4}} \stackrel{P 5,7}{=}$

$\frac{1}{3} \frac{32(\vec{a} \cdot \hat{r}) \vec{a} \cdot \vec{\partial}(\vec{a} \cdot \hat{r})-2|\hat{r} \wedge \vec{a}| \vec{a} \cdot \vec{\partial}|\hat{r} \wedge \vec{a}|}{r^{4}}+$

$\frac{1}{3}\left[3(\vec{a} \cdot \hat{r})^{2}-|\hat{r} \wedge \vec{a}|^{2}\right](-4) \frac{1}{r^{5}} \vec{a} \cdot \vec{\partial} r \stackrel{P 23,25,27}{=}$

$\frac{2(\vec{a} \cdot \hat{r}) \frac{|\hat{r} \wedge \vec{a}|^{2}}{r}+\frac{2}{3}|\hat{r} \wedge \vec{a}| \hat{r} \cdot \vec{a} \frac{|\hat{r} \wedge \vec{a}|}{r}}{r^{4}}-$

$\frac{4}{3}\left[3(\vec{a} \cdot \hat{r})^{2}-|\hat{r} \wedge \vec{a}|^{2}\right] \frac{1}{r^{5}} \vec{a} \cdot \hat{r}=$

$\frac{2(\vec{a} \cdot \hat{r})|\hat{r} \wedge \vec{a}|^{2}+\frac{2}{3}|\hat{r} \wedge \vec{a}|^{2} \hat{r} \cdot \vec{a}-4(\vec{a} \cdot \hat{r})^{3}+}{r^{5}}$

$\frac{\frac{4}{3}|\hat{r} \wedge \vec{a}|^{2} \hat{r} \cdot \vec{a}}{r^{5}}=\frac{-4(\vec{a} \cdot \hat{r})^{3}+4|\hat{r} \wedge \vec{a}|^{2} \vec{a} \cdot \hat{r}}{.}$

Proposition 32

$$
\vec{a} \cdot \vec{\partial} \log r=\frac{\vec{a} \cdot \vec{r}}{r^{2}} .
$$

\section{Proof 32}

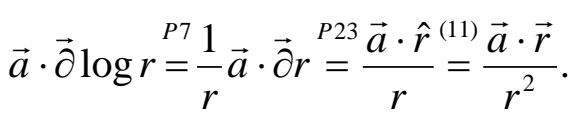

\section{Proposition 33}

For integer $k$ and $\vec{r} \neq 0$ if $k<0$ :

$$
\vec{a} \cdot \vec{\partial} \vec{r}^{2 k}=2 k \vec{a} \cdot \vec{r} \vec{r}^{2(k-1)} .
$$

\section{Proof 33}

$\vec{a} \cdot \vec{\partial} \vec{r}^{2 k}=\vec{a} \cdot \vec{\partial} r^{2 k}=2 k r^{2 k-1} \vec{a} \cdot \vec{\partial} r^{P 23}=2 k r^{2 k-1} \vec{a} \cdot \hat{r}$ $=2 k r^{2 k-2} \vec{a} \cdot \vec{r}=2 k \vec{a} \cdot \vec{r} \vec{r}^{2(k-1)}$.

\section{Proposition 34}

For integer $k$ and $\vec{r} \neq 0$ if $2 k+1<0$ :

$$
\vec{a} \cdot \vec{\partial} \vec{r}^{2 k+1}=\vec{r}^{2 k}(\vec{a}+2 k \vec{a} \cdot \hat{r} \hat{r}) .
$$

\section{Proof 34}

$\vec{a} \cdot \vec{\partial} \vec{r}^{2 k+1}=\vec{a} \cdot \vec{\partial}\left(\vec{r}^{2 k} \vec{r}\right) \stackrel{P 5}{=}\left(\vec{a} \cdot \vec{\partial} \vec{r}^{2 k}\right) \vec{r}+\vec{r}^{2 k} \vec{a} \cdot \vec{\partial} \vec{r}$ $P 8,33$

$\stackrel{P}{=} 2 k \vec{a} \cdot \vec{r}^{2(k-1)} \vec{r}+\vec{r}^{2 k} \vec{a}=\vec{r}^{2 k}(\vec{a}+2 k \vec{a} \cdot \hat{r} \hat{r})$.

Proposition 35 (Taylor expansion of $\frac{1}{\vec{x}-\vec{a}}$ )

$$
\frac{1}{\vec{x}-\vec{a}}=\frac{1}{\vec{x}}+\frac{1}{\vec{x}} \vec{a} \frac{1}{\vec{x}}+\frac{1}{\vec{x}} \vec{a} \frac{1}{\vec{x}} \vec{a} \frac{1}{\vec{x}}+\ldots .
$$

\section{Proof 35}

$\frac{1}{\vec{x}-\vec{a}} \stackrel{P 12}{=} \exp (-\vec{a} \cdot \vec{\partial}) \frac{1}{\vec{x}}=\sum_{k=0}^{\infty} \frac{(-\vec{a} \cdot \vec{\partial})^{k}}{k !} \frac{1}{\vec{x}}$,

$(-\vec{a} \cdot \vec{\partial})^{k} \frac{1}{\vec{x}}=(-\vec{a} \cdot \vec{\partial})^{k-1}(-\vec{a} \cdot \vec{\partial}) \frac{1}{\vec{x}}=$

$(-\vec{a} \cdot \vec{\partial})^{k-1} \frac{1}{\vec{x}} \vec{a} \frac{1}{\vec{x}}=(-\vec{a} \cdot \vec{\partial})^{k-2}(-\vec{a} \cdot \vec{\partial})\left[\frac{1}{\vec{x}} \vec{a} \frac{1}{\vec{x}}\right]^{P 5}=$

$(-\vec{a} \cdot \vec{\partial})^{k-2}\left[\left(-\vec{a} \cdot \vec{\partial} \frac{1}{\vec{x}}\right) \vec{a} \frac{1}{\vec{x}}+\frac{1}{\vec{x}} \vec{a}\left(-\vec{a} \cdot \vec{\partial} \frac{1}{\vec{x}}\right)\right] \stackrel{P 28}{=}$

$2(-\vec{a} \cdot \vec{\partial})^{k-2} \frac{1}{\vec{x}} \vec{a} \frac{1}{\vec{x}} \vec{a} \frac{1}{\vec{x}} \stackrel{P 5,28}{=}$

$23(-\vec{a} \cdot \vec{\partial})^{k-3} \frac{1}{\vec{x}} \vec{a} \frac{1}{\vec{x}} \vec{a} \frac{1}{\vec{x}} \vec{a} \frac{1}{\vec{x}} \stackrel{P 5,28}{=} \ldots=$

$k !(-\vec{a} \cdot \vec{\partial})^{k-k} \frac{1}{\vec{x}}\left(\vec{a} \frac{1}{\vec{x}}\right)^{k}=k ! \frac{1}{\vec{x}}\left(\vec{a} \frac{1}{\vec{x}}\right)^{k}$

$\Rightarrow \frac{1}{k !}(-\vec{a} \cdot \vec{\partial}) \frac{1}{\vec{x}}=\frac{1}{\vec{x}}\left(\vec{a} \frac{1}{\vec{x}}\right)^{k}$

$\Rightarrow \frac{1}{\vec{x}-\vec{a}}=\sum_{k=0}^{\infty} \frac{1}{\vec{x}}\left(\vec{a} \frac{1}{\vec{x}}\right)^{k}$.

Proposition 36 (Legendre Polynomials)

The Legendre Polynomials $P_{n}$ are defined by:

$$
\frac{1}{|\vec{x}-\vec{a}|} \equiv \sum_{n=0}^{\infty} \frac{P_{n}(\hat{x} \vec{a})}{|\vec{x}|^{n+1}}=\sum_{n=0}^{\infty} \frac{P_{n}(\vec{x} \vec{a})}{|\vec{x}|^{2 n+1}} .
$$

The explicit first four polynomials are:

$$
\begin{aligned}
& P_{0}(\vec{x} \vec{a})=1 \\
& P_{1}(\vec{x} \vec{a})=\vec{x} \cdot \vec{a} \\
& P_{2}(\vec{x} \vec{a})=\frac{1}{2}\left[3(\vec{x} \cdot \vec{a})^{2}-\vec{a}^{2} \vec{x}^{2}\right] \\
& =(\vec{x} \cdot \vec{a})^{2}+\frac{1}{2}(\vec{x} \wedge \vec{a})^{2} \\
& P_{3}(\vec{x} \vec{a})=\frac{1}{2}\left[5(\vec{x} \cdot \vec{a})^{3}-3 \vec{a}^{2} \vec{x}^{2} \vec{x} \cdot \vec{a}\right]= \\
& (\vec{x} \cdot \vec{a})^{3}+\frac{3}{2} \vec{x} \cdot \vec{a}(\vec{x} \wedge \vec{a})^{2}
\end{aligned}
$$


$P_{n}(\vec{x} \vec{a})=|\vec{x}|^{n} P_{n}(\hat{x} \vec{a})=|\vec{x}|^{n}|\vec{a}|^{n} P_{n}(\hat{x} \hat{a})$.

\section{Proof 36}

$F(\vec{x}-\vec{a})=\frac{1}{|\vec{x}-\vec{a}|} \stackrel{P 12}{=} \exp (-\vec{a} \cdot \vec{\partial}) F(\vec{x})=$

$\sum_{n=0}^{\infty} \frac{(-\vec{a} \cdot \vec{\partial})}{n !} \frac{1}{|\vec{x}|}=$

$\frac{1}{|\vec{x}|}-\vec{a} \cdot \vec{\partial} \frac{1}{|\vec{x}|}+\frac{1}{2}(\vec{a} \cdot \vec{\partial})^{2} \frac{1}{|\vec{x}|}-\frac{1}{6}(\vec{a} \cdot \vec{\partial})^{3} \frac{1}{|\vec{x}|}+\ldots$

$\Rightarrow P_{0}(\vec{x} \vec{a})=1$,

$-\vec{a} \cdot \vec{\partial} \frac{1}{|\vec{x}|}=\frac{1}{|\vec{x}|^{2}} \vec{a} \cdot \vec{\partial}|\vec{x}|^{P 10}=\frac{1}{|\vec{x}|^{2}} \vec{a} \cdot \hat{x}=\frac{\vec{a} \cdot \vec{x}}{|\vec{x}|^{3}}$

$\Rightarrow P_{1}(\vec{x} \vec{a})=\vec{x} \cdot \vec{a}$,

$\frac{1}{2}(\vec{a} \cdot \vec{\partial})^{2} \frac{1}{|\vec{x}|}=-\frac{1}{2}(\vec{a} \cdot \vec{\partial})(-\vec{a} \cdot \vec{\partial}) \frac{1}{|\vec{x}|}=$

$-\frac{1}{2}(\vec{a} \cdot \vec{\partial}) \frac{\vec{a} \cdot \vec{x}}{|\vec{x}|^{35}}=$

$-\frac{1}{2}\left[\vec{a} \cdot \vec{\partial} \frac{1}{|\vec{x}|^{3}}\right] \vec{a} \cdot \vec{x}-\frac{1}{2} \frac{1}{|\vec{x}|^{3}} \vec{a} \cdot \vec{\partial}(\vec{a} \cdot \vec{x}) \stackrel{P 7,21}{=}$

$-\frac{1}{2}\left[\frac{-3}{|\vec{x}|^{4}} \vec{a} \cdot \vec{\partial}|\vec{x}|\right] \vec{a} \cdot \vec{x}-\frac{1}{2} \frac{1}{|\vec{x}|^{3}} \vec{a} \cdot \vec{a}=$

$\frac{1}{2} \frac{3}{|\vec{x}|^{4}} \vec{a} \cdot \hat{x}(\vec{a} \cdot \vec{x})-\frac{1}{2} \frac{1}{|\vec{x}|^{3}} \vec{a}^{2} \underset{(5)}{(11)}$

$\frac{1}{2} \frac{3(\vec{a} \cdot \vec{x})^{2}-\vec{x}^{2} \vec{a}^{2}}{|\vec{x}|^{5}}=\frac{(\vec{a} \cdot \vec{x})^{2}+\frac{1}{2}\left\{(\vec{a} \cdot \vec{x})^{2}-\vec{x}^{2} \vec{a}^{2}\right\}}{|\vec{x}|^{5}}$

$=\frac{(\vec{a} \cdot \vec{x})^{2}+\frac{1}{2}(\vec{a} \wedge \vec{x})^{2}}{|\vec{x}|^{5}}$

$\Rightarrow P_{2}(\vec{x} \vec{a})=\frac{1}{2}\left[3(\vec{x} \cdot \vec{a})^{2}-\vec{a}^{2} \vec{x}^{2}\right]$

$=(\vec{x} \cdot \vec{a})^{2}+\frac{1}{2}(\vec{x} \wedge \vec{a})^{2}$,

$-\frac{1}{6}(\vec{a} \cdot \vec{\partial})^{3} \frac{1}{|\vec{x}|}=-\frac{1}{3}(\vec{a} \cdot \vec{\partial})\left\{\frac{1}{2}(\vec{a} \cdot \vec{\partial})^{2} \frac{1}{|\vec{x}|}\right\}=$

$-\frac{1}{3}(\vec{a} \cdot \vec{\partial})\left\{\frac{1}{2} \frac{3(\vec{a} \cdot \vec{x})^{2}-\vec{x}^{2} \vec{a}^{2}}{|\vec{x}|^{5}}\right\}^{P 4,5}=$ $=-\frac{1}{6}\left(\vec{a} \cdot \vec{\partial} \frac{1}{|\vec{x}|^{5}}\right)\left\{3(\vec{a} \cdot \vec{x})^{2}-\vec{x}^{2} \vec{a}^{2}\right\}$

$-\frac{1}{6|\vec{x}|^{5}}\left\{32(\vec{a} \cdot \vec{x}) \vec{a} \cdot \vec{\partial}(\vec{a} \cdot \vec{x})-\vec{a}^{2}(\vec{a} \cdot \vec{\partial}) \vec{x}^{2}\right\} \stackrel{P 5,7,21,23}{=}$

$-\frac{-5}{6|\vec{x}|^{6}}(\vec{a} \cdot \vec{\partial}|\vec{x}|)\left\{3(\vec{a} \cdot \vec{x})^{2}-\vec{x}^{2} \vec{a}^{2}\right\}$

$-\frac{1}{6|\vec{x}|^{5}}\left\{6(\vec{a} \cdot \vec{x}) \vec{a} \cdot \vec{a}-\vec{a}^{2} 2(\vec{a} \cdot \vec{x})\right\}^{P 10}=$

$\frac{5}{6|\vec{x}|^{6}} \frac{\vec{a} \cdot \vec{x}}{|\vec{x}|}\left\{3(\vec{a} \cdot \vec{x})^{2}-\vec{x}^{2} \vec{a}^{2}\right\}-\frac{4 \vec{a}^{2}(\vec{a} \cdot \vec{x})}{6|\vec{x}|^{5}}=$

$\frac{15(\vec{a} \cdot \vec{x})^{3}-5 \vec{x}^{2} \vec{a}^{2} \vec{a} \cdot \vec{x}-4 \vec{x}^{2} \vec{a}^{2}(\vec{a} \cdot \vec{x})}{6|\vec{x}|^{7}}=$

$\frac{1}{2|\vec{x}|^{7}}\left[5(\vec{a} \cdot \vec{x})^{3}-3 \vec{x}^{2} \vec{a}^{2} \vec{a} \cdot \vec{x}\right]=$

$\left.\frac{1}{|\vec{x}|^{7}}\left[(\vec{a} \cdot \vec{x})^{3}+\frac{3}{2} \vec{a} \cdot \vec{x}\left\{(\vec{a} \cdot \vec{x})^{2}-\vec{x}^{2} \vec{a}^{2}\right\}\right]\right]_{=}^{(43)}$

$\frac{1}{|\vec{x}|^{7}}\left[(\vec{a} \cdot \vec{x})^{3}+\frac{3}{2} \vec{a} \cdot \vec{x}(\vec{a} \wedge \vec{x})^{2}\right]$

$\Rightarrow P_{3}(\vec{x} \vec{a})=\frac{1}{2}\left[5(\vec{x} \cdot \vec{a})^{3}-3 \vec{a}^{2} \vec{x}^{2} \vec{x} \cdot \vec{a}\right]=$

$(\vec{x} \cdot \vec{a})^{3}+\frac{3}{2} \vec{x} \cdot \vec{a}(\vec{x} \wedge \vec{a})^{2}$

Homogeneity of degree $n$ of the $P_{n}$ :

$\frac{(-\vec{a} \cdot \vec{\partial})^{n}}{n !} \frac{1}{|\vec{x}|} \stackrel{\text { def }}{=} \frac{P_{n}(\vec{x} \vec{a})}{|x|^{2 n+1}}$

$\Rightarrow(-\vec{a} \cdot \vec{\partial})^{n} \frac{1}{|\vec{x}|}=n ! \frac{P_{n}(\vec{x} \vec{a})}{|x|^{2 n+1}}$ and

$(-\vec{a} \cdot \vec{\partial})^{n+1} \frac{1}{|\vec{x}|}=(n+1) ! \frac{P_{n+1}(\vec{x} \vec{a})}{|x|^{2(n+1)+1}}$

$\Rightarrow \frac{(n+1) !}{n !} \frac{P_{n+1}(\vec{x} \vec{a})}{|\vec{x}|^{2(n+1)+1}}=(-\vec{a} \cdot \vec{\partial}) \frac{P_{n}(\vec{x} \vec{a})}{|\vec{x}|^{2 n+1}}=$

$\left(-\vec{a} \cdot \vec{\partial} \frac{1}{|\vec{x}|^{2 n+1}}\right) P_{n}(\vec{x} \vec{a})-\frac{1}{|\vec{x}|^{2 n+1}} \vec{a} \cdot \vec{\partial} P_{n}(\vec{x} \vec{a})=$

$\frac{2 n+1}{|\vec{x}|^{2 n+2}} P_{n}(\vec{x} \vec{a}) \vec{a} \cdot \vec{\partial}|\vec{x}|-\frac{1}{|\vec{x}|^{2 n+1}} \vec{a} \cdot \vec{\partial} P_{n}(\vec{x} \vec{a}) \stackrel{P 10}{=}$

$\frac{2 n+1}{|\vec{x}|^{2 n+2}} P_{n}(\vec{x} \vec{a}) \frac{\vec{a} \cdot \vec{x}}{|\vec{x}|}-\frac{1}{|\vec{x}|^{2 n+1}} \vec{a} \cdot \vec{\partial} P_{n}(\vec{x} \vec{a})=$ 
$=\frac{2 n+1}{|\vec{x}|^{2 n+3}} P_{n}(\vec{x} \vec{a}) \vec{a} \cdot \vec{x}-\frac{1}{|\vec{x}|^{2 n+1}} \vec{a} \cdot \vec{\partial} P_{n}(\vec{x} \vec{a})$.

$P_{n}(\vec{x} \vec{a}) \vec{a} \cdot \vec{x}$ is a homogeneous function of degree $n+1$, if we assume $P_{n}$ to be homogeneous of degree $n$ :

$P_{n}(\vec{x} \vec{a}) \stackrel{\text { assume }}{=} \sum_{k=0}^{n} \alpha_{k}(\vec{x} \cdot \vec{a})^{k}|\vec{x}|^{n-k} \vec{a}^{n-k}$, $\alpha_{k}=$ const. which is especially true for $n=0,1,2,3$. The right term

$\vec{a} \cdot \vec{\partial} P_{n}(\vec{x} \vec{a})=\vec{a} \cdot \vec{\partial} \sum_{k=0}^{n} \alpha_{k}(\vec{x} \cdot \vec{a})^{k}|\vec{x}|^{n-k} \vec{a}^{n-k} \stackrel{P 4,5,9}{=}$

$\sum_{k=0}^{n} \alpha_{k}\left\{\vec{a} \cdot \vec{\partial}(\vec{x} \cdot \vec{a})^{k}|\vec{x}|^{n-k} \vec{a}^{n-k}+\right.$

$\alpha_{k}(\vec{x} \cdot \vec{a})^{k}\left\{\vec{a} \cdot \vec{\partial}|\vec{x}|^{n-k} \int_{a}^{n-k} \stackrel{P 5,7}{=}\right.$

$\sum_{k=0}^{n} \alpha_{k}\left\{k(\vec{x} \cdot \vec{a})^{k-1} \vec{a} \cdot \vec{\partial}(\vec{x} \cdot \vec{a})\right\}|\vec{x}|^{n-k} \vec{a}^{n-k}+$

$\alpha_{k}(\vec{x} \cdot \vec{a})^{k}\left\{(n-k)|\vec{x}|^{n-k-1} \vec{a} \cdot \vec{\partial}|\vec{x}| \mid \vec{a}^{n-k} \stackrel{P 10,21}{=}\right.$

$\left.\sum_{k=0}^{n} \alpha_{k}\left\{k(\vec{x} \cdot \vec{a})^{k-1} \vec{a} \cdot \vec{a}\right)\right\}|\vec{x}|^{n-k} \vec{a}^{n-k}+$

$\alpha_{k}(\vec{x} \cdot \vec{a})^{k}\left\{(n-k)|\vec{x}|^{n-k-1} \frac{\vec{a} \cdot \vec{x}}{|\vec{x}|} \vec{a}^{n-k}=\right.$

$\sum_{k=0}^{n} \alpha_{k} k(\vec{x} \cdot \vec{a})^{k-1} \frac{|\vec{x}|^{2}}{|\vec{x}|^{2}}|\vec{x}|^{n-k} \vec{a}^{2} \vec{a}^{n-k}+$

$\alpha_{k}(n-k)(\vec{x} \cdot \vec{a})^{k+1} \frac{|\vec{x}|^{n-k-1}}{|\vec{x}|} \frac{|\vec{x}|}{|\vec{x}|} \vec{a}^{n-k}=$

$\frac{1}{|\vec{x}|^{2}} \sum_{k=0}^{n} \alpha_{k} k(\vec{x} \cdot \vec{a})^{k-1}|\vec{x}|^{n-k+2} \vec{a}^{n-k+2}+$

$\alpha_{k}(n-k)(\vec{x} \cdot \vec{a})^{k+1}|\vec{x}|^{n-k} \vec{a}^{n-k}$

yields $|\vec{x}|^{2} \vec{a} \cdot \vec{\partial} P_{n}(\vec{x} \vec{a})$, to be homogeneous of degree $n+1$.

Hence $\frac{(n+1) !}{n !} \frac{P_{n+1}(\vec{x} \vec{a})}{|\vec{x}|^{(n+1)+1}}=$

(Polynomial homogeneous of degree $n+1)$

$|\vec{x}|^{2(n+1)+1}$. By induction

every $P_{n}$ will therefore be homogeneous of degree $n$. This and

the explicit expressions above for $\frac{P_{n+1}(\vec{x} \vec{a})}{|\vec{x}|^{2(n+1)+1}}$ fully prove for all $n: \quad P_{n}(\vec{x} \vec{a})=|\vec{x}|^{n} P_{n}(\hat{x} \vec{a})=|\vec{x}|^{n}|\vec{a}|^{n} P_{n}(\hat{x} \hat{a})$.

Definition 37 (redefinition of differential, over-dots)

$$
\underline{F}(\vec{a})=\vec{a} \cdot \vec{\partial} F \stackrel{(7)}{=} \frac{1}{2}(\vec{a} \vec{\partial} F+\dot{\vec{\partial}} \vec{a} \dot{F}),
$$

where the over-dots indicate, that only $F$ is to be differentiated and not $\vec{a}$.

\section{Proposition 38}

For $\vec{a} \notin A_{n} \equiv G^{1}(I), P=P_{I}$ :

$$
\vec{a} \cdot \vec{\partial}_{\vec{x}}=\vec{a} \cdot P\left(\vec{\partial}_{\vec{x}}\right)=P(\vec{a}) \cdot \vec{\partial}_{\vec{x}}, P(\vec{a}) \in A_{n} .
$$

\section{Proof 38}

$\vec{a} \cdot \vec{\partial}_{\vec{x}}=\vec{a} \cdot P\left(\vec{\partial}_{\vec{x}}\right)=\sum_{k=1}^{P 18} \vec{a} \cdot \vec{a}^{k}\left(\vec{a}_{k} \cdot \vec{\partial}_{\vec{x}}\right)=$
$\sum_{k=1}^{n} P(\vec{a}) \cdot \vec{a}^{k}\left(\vec{a}_{k} \cdot \vec{\partial}_{\vec{x}}\right)=P(\vec{a}) \cdot \vec{\partial}_{\vec{x}}$.

\section{Proposition 39}

$$
\begin{gathered}
\underline{F}(\vec{a})=\underline{F}(P(\vec{a}))=P(\vec{a}) \cdot \vec{\partial} F . \\
\underline{F}(\vec{a})=0, \text { if } P(\vec{a})=0 .
\end{gathered}
$$

\section{Proof 39}

$$
\begin{aligned}
& \underline{F}(\vec{a}) \stackrel{\text { def } 13, P 38}{=} \underline{F}(P(\vec{a})) \stackrel{\text { def } 13}{=} P(\vec{a}) \cdot \vec{\partial} F, \\
& \Rightarrow \underline{F}(\vec{a})=0, \text { if } P(\vec{a})=0 .
\end{aligned}
$$

\section{Proposition 40 (differential of composite functions)}

For $F(\vec{x})=G(f(\vec{x}))$ and

$$
\begin{gathered}
f: \vec{x} \in A_{n}=G^{1}(I) \rightarrow f(\vec{x}) \in A_{n}^{\prime}=G^{1}\left(I^{\prime}\right) \\
\vec{a} \cdot \vec{\partial} F=\underline{f(\vec{a}) \cdot \vec{\partial} G} \\
\underline{F}(\vec{a})=\underline{G}(\underline{f}(\vec{a})) \text { (Def. 13) }
\end{gathered}
$$

The differential of composite functions is the composite of differentials.

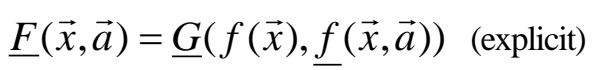

\section{Proof 40}

Taylor expansion (P12):

$f(\vec{x}+\tau \vec{a})=f(\vec{x})+\tau \vec{a} \cdot \vec{\partial} f(\vec{x})+\frac{1}{2} \tau^{2}(\vec{a} \cdot \vec{\partial})^{2} f(\vec{x})+\ldots$

$\left.\vec{a} \cdot \vec{\partial} G(f(\vec{x})) \stackrel{\text { def } 1}{=} \frac{d}{d \tau} G(f(\vec{x}+\tau \vec{a}))\right|_{\tau=0} \begin{gathered}\text { Taylor(P12) } \\ = \\ \operatorname{def} 13\end{gathered}$ 
$=\left.\left.\frac{d}{d \tau} G(f(\vec{x})+\tau \underline{f}(\vec{a}))\right|_{\tau=0} \stackrel{\operatorname{def} 1}{=} \underline{f}(\vec{a}) \cdot \vec{\partial}_{\vec{x}^{\prime}} G\left(\vec{x}^{\prime}\right)\right|_{\vec{x}^{\prime}=f(\vec{x})}$

$=\underline{f}(\vec{a}) \cdot \vec{\partial} G$ (evaluation at corresponding points.)

Definition 41 (second differential)

$$
F_{\vec{a} \vec{b}}(\vec{x}) \equiv \vec{b} \cdot \dot{\vec{\partial}} \vec{a} \cdot \vec{\partial} \dot{F}(\vec{x}) .
$$

Suppressing $\vec{x}: F_{\vec{a} \vec{b}} \equiv \vec{b} \cdot \dot{\vec{\partial}} \vec{a} \cdot \vec{\partial} \dot{F}$.

Proposition 42 (integrability condition)

$$
F_{\vec{a} \vec{b}}=F_{\vec{b} \vec{a}} .
$$

The second differential is a symmetric bilinear function of its differential arguments $\vec{a}, \vec{b}$.

\section{Proof 42}

$F_{\vec{a} \vec{b}}(\vec{x}) \equiv \vec{b} \cdot \dot{\vec{\partial}} \vec{a} \cdot \vec{\partial} \dot{F}(\vec{x})=\overrightarrow{d e f}=\left.\vec{b} \cdot \dot{\vec{\partial}} \frac{d \dot{F}(\vec{x}+\tau \vec{a})}{d \tau}\right|_{\tau=0}=$ $\left.\frac{d^{2} F(\vec{x}+\tau \vec{a}+\sigma \vec{b})}{d \sigma d \tau}\right|_{\substack{\tau=0 \\ \sigma=0}}=\lim _{\sigma \rightarrow 0} \lim _{\tau \rightarrow 0}$

$\frac{\frac{F(\vec{x}+\tau \vec{a}+\sigma \vec{b})-F(\vec{x}+\sigma \vec{b})}{\tau}-\frac{F(\vec{x}+\tau \vec{a})-F(\vec{x})}{\tau}}{\sigma}$

\section{$\sigma$}

which is symmetric under $(\vec{a}, \tau) \leftrightarrow(\vec{b}, \sigma)$. Hence

$$
F_{\vec{a} \vec{b}}(\vec{x}) \equiv \vec{b} \cdot \dot{\vec{\partial}} \vec{a} \cdot \vec{\partial} \dot{F}(\vec{x})=\vec{a} \cdot \dot{\vec{\partial}} \vec{b} \cdot \vec{\partial} \dot{F}(\vec{x})=F_{\vec{b} \vec{a}}(\vec{x}) .
$$

The bilinearity follows from the linearity in each argument (P2, P3 and P14).

Proposition 43 (differential of identity function)

$$
\vec{a} \cdot \vec{\partial}_{\vec{x}} \vec{x}=P(\vec{a})=\vec{\partial}_{\vec{x}}(\vec{x} \cdot \vec{a})
$$

\section{Proof 43}

$\vec{a} \cdot \vec{\partial}_{\vec{x}} \vec{x}=P_{I}(\vec{a}) \cdot \vec{\partial}_{\vec{x}} \vec{x}=P_{I}(\vec{a}) \quad$ (first identity).

Especially for base vectors $\vec{a}_{k} \in A_{n}=G^{1}(I)$ :

$$
\begin{gathered}
\vec{a}_{k} \cdot \vec{\partial}_{\vec{x}} \vec{x}=P_{I}\left(\vec{a}_{k}\right)=\vec{a}_{k}, \\
\vec{\partial}_{\vec{x}}(\vec{x} \cdot \vec{a})=\sum_{k} \vec{a}^{k} \vec{a}_{k} \cdot \vec{\partial}_{\vec{x}}(\vec{x} \cdot \vec{a})=\sum_{k} \vec{a}^{k} \vec{a}_{k} \cdot \vec{a} \\
=P_{I}(\vec{a}) \text { (second identity). }
\end{gathered}
$$

Proposition 44 (operator identity)

$$
\vec{\partial}_{\vec{x}}=P_{I}\left(\vec{\partial}_{\vec{x}}\right)=\vec{\partial}_{\vec{a}} \vec{a} \cdot \vec{\partial}_{\vec{x}} \cdot
$$

Proof 44
Propositions 18 and 43.

Proposition 45 (derivative from differential)

$$
\vec{\partial}_{\vec{x}} F(\vec{x})=\vec{\partial}_{\vec{a}} \vec{a} \cdot \vec{\partial}_{\vec{x}} F(\vec{x})=\vec{\partial}_{\vec{a}} F(\vec{x}, \vec{a}) .
$$

\section{Proof 45}

Proposition 44 and definition 13.

\section{Definition 46}

$$
\vec{\partial} F=\vec{\partial}_{\vec{x}} F(\vec{x}) \stackrel{P 45}{=} \vec{\partial}_{\vec{a}} \underline{F}(\vec{x}, \vec{a})=\underline{\vec{\partial}} \underline{F},
$$

where $\underline{\vec{\partial}}$ is the derivative with respect to the differential argument $\vec{a}$ of $\underline{F}(\vec{x}, \vec{a})$.

\section{Proposition 47}

$$
\vec{\partial} F=\vec{\partial} \cdot F+\vec{\partial} \wedge F .
$$

\section{Proof 47}

Vector property (P. 18) of $\vec{\partial}=\vec{\partial}_{\vec{x}}$ and (18).

\section{Proposition 48 (gradient)}

For scalar $F=\Phi(\vec{x})$ :

$$
\vec{\partial} \cdot \Phi=0, \vec{\partial} \Phi=\vec{\partial} \wedge \Phi=\dot{\Phi} \dot{\vec{\partial}}
$$

\section{Proof 48}

(21), P. 47 and (23).

\section{Remark 49}

In proposition 48 the special definition of Hestenes and Sobczyk[2] in (20) and (21) for the inner product becomes important. It should be possible to make it more intuitive by replacing the inner product with the contraction [4].

\section{Definition 50}

Divergence of $F: \vec{\partial} \cdot F$,

Curl of $F: \quad \vec{\partial} \wedge F$

(Full vector derivative of $F: \vec{\partial} F$.)

Proposition 51 (vector derivative of sums)

$$
\vec{\partial}(F+G)=\vec{\partial} F+\vec{\partial} G .
$$

\section{Proof 51}

$$
\begin{aligned}
& \vec{\partial}(F+G) \stackrel{\text { def } 46}{=} \vec{\partial}(\underline{F+G)} \stackrel{P 4}{=} \underline{\partial}(\underline{F}+\underline{G}) \stackrel{P 18}{=} \\
& \sum_{k} \vec{a}^{k} \vec{a}_{k} \cdot \vec{\partial}_{\vec{a}}(\underline{F}+\underline{G})=
\end{aligned}
$$$$
\sum_{k} \vec{a}^{k}\left(\vec{a}_{k} \cdot \vec{\partial}_{\vec{a}} \underline{F}+\vec{a}_{k} \cdot \vec{\partial}_{\vec{a}} \underline{G}\right) \underset{(19)}{\text { distributivity }}
$$$$
\sum_{k} \vec{a}^{k} \vec{a}_{k} \cdot \vec{\partial}_{\vec{a}} \underline{F}+\sum_{k} \vec{a}^{k} \vec{a}_{k} \cdot \vec{\partial}_{\vec{a}} \underline{\operatorname{Gef} 46} \underset{\operatorname{P18}}{=} \vec{\partial} F+\vec{\partial} G .
$$

Note that, geometric multiplication is distributive with respect 
to addition.

Proposition 52 (vector derivative of products)

$$
\vec{\partial}(F G)=\dot{\vec{\partial}} \dot{F} G+\dot{\vec{\partial}} F \dot{G}
$$

\section{Proof 52}

$\vec{\partial}(F G) \stackrel{\text { def } 46}{=} \vec{\partial}(\underline{F G)})^{P 5} \vec{\partial}(\underline{F G}+F \underline{G}) \underset{P 51}{\stackrel{\text { def } 46}{=}}$

$\underline{\vec{\partial}} \underline{F} G+\underline{\vec{\partial}} F \underline{G} \stackrel{\operatorname{def}}{=46} \dot{\vec{\partial}} \dot{F} G+\dot{\vec{\partial}} F \dot{G}$.

The third equality is a special case of P. 51, if we take the definition of $\vec{\partial}$ in Def. 46 into account. The last term is to be interpreted as: $\dot{\vec{\partial}} F \dot{G}=\vec{\partial}_{\vec{y}}\left(\left.F(\vec{x}) G(\vec{y})\right|_{\vec{y}=\vec{x}}\right.$.

\section{Proposition 53}

$$
\vec{\partial} \vec{x}^{2}=2 \vec{x}
$$

\section{Proof 53}

$\vec{\partial} \vec{x}^{2}=(\vec{\partial} \vec{x}) \vec{x}+\dot{\vec{\partial}} \vec{x} \dot{\vec{x}}^{P 18}=$

$\left(\sum_{k} \vec{a}^{k} \vec{a}_{k} \cdot \vec{\partial}_{\vec{x}} \vec{x}\right) \vec{x}+\sum_{k} \vec{a}^{k} \vec{x} \vec{a}_{k} \cdot \vec{\partial}_{\vec{x}} \vec{x}=$

$\left(\sum_{k} \vec{a}^{k} \vec{a}_{k}\right) \vec{x}+\sum_{k} \vec{a}^{k} \vec{x} \vec{a}_{k}{ }^{(7)}=2 \sum_{k} \vec{a}^{k} \vec{x} \cdot \vec{a}_{k} \stackrel{(32)}{=} 2 \vec{x}$.

Proposition 54

$$
\vec{\partial}|\vec{x}|=\hat{x}
$$

\section{Proof 54}

$\vec{\partial}|\vec{x}|=\sum_{k} \vec{a}^{k} \vec{a}_{k} \cdot \vec{\partial}_{\vec{x}}|\vec{x}|^{P 10}=\sum_{k} \vec{a}^{k} \vec{a}_{k} \cdot \hat{x} \stackrel{(32)}{=} \hat{x}$.

\section{Proposition 55}

For $F=F(|\vec{x}|): \quad \vec{\partial} F=\hat{x} \frac{d F}{d|\vec{x}|}$.

\section{Proof 55}

$\vec{a} \cdot \vec{\partial} F \stackrel{P 7}{=} \vec{a} \cdot \vec{\partial}|\vec{x}| \frac{d F}{d|\vec{x}|}=\vec{a} \cdot \hat{x} \frac{d F}{d|\vec{x}|}$

$\Rightarrow \vec{\partial} F \stackrel{d e f}{=} \vec{\partial}_{\vec{a}} \vec{a} \cdot \vec{\partial} F=\vec{\partial}_{\vec{a}} \vec{a} \cdot \hat{x} \frac{d F}{d|\vec{x}|}=\hat{x} \frac{d F}{d|\vec{x}|}$.

Definition 56 (sides of differentiation)

Only right side differentiation:

$$
F \vec{\partial} G \stackrel{\text { def } 46}{=} F \underline{\partial} \underline{G}=F \dot{\vec{\partial}} \dot{G} .
$$

Left and right side differentiation (another form of the product rule P. 52):

$$
\dot{F} \dot{\vec{\partial}} \dot{G}=\dot{F} \dot{\vec{\partial}} G+F \dot{\vec{\partial}} \dot{G} .
$$

For $f: \vec{x} \in A_{n}=G^{1}(I) \rightarrow f(\vec{x}) \in A_{n}^{\prime}=G^{1}\left(I^{\prime}\right)$

$$
\bar{f}\left(\vec{a}^{\prime}\right) \equiv \underline{\vec{\partial}}\left(\underline{f} \cdot \vec{a}^{\prime}\right),
$$

is the adjoint of $f$ or explicitly:

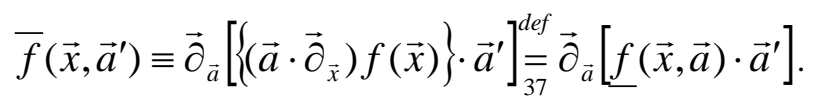

\section{Proposition 58}

$$
\bar{f}\left(\vec{a}^{\prime}\right)=\vec{\partial}\left(f \cdot \vec{a}^{\prime}\right),
$$

or explicitly:

$$
\bar{f}\left(\vec{x}, \vec{a}^{\prime}\right)=\vec{\partial}_{\vec{x}}\left(f(\vec{x}) \cdot \vec{a}^{\prime}\right) .
$$

\section{Proof 58}

$$
\begin{aligned}
& \bar{f}\left(\vec{x}, \vec{a}^{\prime}\right) \stackrel{\text { def } 57}{=} \vec{\partial}_{\vec{a}}\left[\left\{\left(\vec{a} \cdot \vec{\partial}_{\vec{x}}\right) f(\vec{x})\right\} \cdot \vec{a}^{\prime}\right]= \\
& \vec{\partial}_{\vec{a}}\left(\vec{a} \cdot \vec{\partial}_{\vec{x}}\right)\left[f(\vec{x}) \cdot \vec{a}^{\prime}\right]^{P 45} \vec{\partial}_{\vec{x}}\left(f(\vec{x}) \cdot \vec{a}^{\prime}\right) . \\
& \text { ([2] p. 50; [5] p. } 23(1.109), \text { p. } 24(1.118), \text { p. } 104 \text { (5.11).) }
\end{aligned}
$$

\section{Proposition 59}

$$
\begin{aligned}
& \bar{f}\left(\vec{a}^{\prime}+\vec{b}^{\prime}\right)=\bar{f}\left(\vec{a}^{\prime}\right)+\bar{f}\left(\vec{b}^{\prime}\right) . \\
& \bar{f}\left(\alpha \vec{a}^{\prime}\right)=\alpha \bar{f}\left(\vec{a}^{\prime}\right), \alpha \text { scalar. }
\end{aligned}
$$

\section{Proof 59}

Linearity of the inner product (9).

\section{Proposition 60}

$$
P\left(\bar{f}\left(\vec{a}^{\prime}\right)\right)=\bar{f}\left(\vec{a}^{\prime}\right) .
$$

\section{Proof 60}

$$
\begin{aligned}
& P\left(\bar{f}\left(\vec{a}^{\prime}\right)\right)^{\text {def } 57, P 58}=P(\vec{\partial}_{\vec{x}} \underbrace{\left(f(\vec{x}) \cdot \vec{a}^{\prime}\right)}_{\text {scalar }})= \\
& P\left(\vec{\partial}_{\vec{x}}\right) f(\vec{x}) \cdot \vec{a}^{\prime}=\vec{\partial}_{\vec{x}}\left(f(\vec{x}) \cdot \vec{a}^{\prime}\right)=\bar{f}\left(\vec{a}^{\prime}\right) .
\end{aligned}
$$

\section{Proposition 61}

$$
\bar{f}\left(\vec{a}^{\prime}\right)=\vec{\partial}_{\bar{a}}\left(\underline{f}(\vec{a}) \cdot \vec{a}^{\prime}\right)=\bar{f}\left(P^{\prime}\left(\vec{a}^{\prime}\right)\right),
$$

with $P^{\prime}$ the projection into the range of $f$ and $f$, i.e. into $A_{n}^{\prime} \equiv G^{1}\left(I^{\prime}\right)$

\section{Proof 61}

$\bar{f}\left(\vec{a}^{\prime}\right) \stackrel{\text { def } 57}{=} \vec{\partial}_{\vec{a}}\left(\underline{f}(\vec{a}) \cdot \vec{a}^{\prime}\right) \stackrel{P 58}{=} \vec{\partial}_{\vec{x}}\left(f(\vec{x}) \cdot \vec{a}^{\prime}\right)=$ $\vec{\partial}_{\vec{x}}\left(P^{\prime} f(\vec{x}) \cdot \vec{a}^{\prime}\right)=\vec{\partial}_{\vec{x}}\left(f(\vec{x}) \cdot P^{\prime}\left(\vec{a}^{\prime}\right)\right)=\stackrel{P 58}{=} \bar{f}\left(P^{\prime}\left(\vec{a}^{\prime}\right)\right)$.

Proposition 62

$$
P \bar{f} P^{\prime}\left(\vec{a}^{\prime}\right)=\bar{f}\left(\vec{a}^{\prime}\right),
$$




$$
P^{\prime} \underline{f} P(\vec{a})=\underline{f}(\vec{a})
$$

\section{Proof 62}

Line 1: Propositions 57,58.

Line 2: P. 39 and because the range of $f$ and $f$ is $A_{n}^{\prime}=G^{1}\left(I^{\prime}\right)$.

Proposition 63 (change of variables)

For $F(\vec{x})=G(f(\vec{x}))$, i.e. $\vec{x} \rightarrow \vec{x}^{\prime}=f(\vec{x})$ :

$$
\vec{\partial}_{\vec{x}} F(\vec{x})=\bar{f}\left(\vec{\partial}_{\vec{x}^{\prime}}\right) G\left(\vec{x}^{\prime}\right) \text {, i.e. } \vec{\partial}_{\vec{x}}=\bar{f}\left(\vec{\partial}_{\vec{x}^{\prime}}\right) .
$$

\section{Proof 63}

$\vec{\partial}_{\vec{x}} F(\vec{x})={ }^{P 44} \vec{\partial}_{\vec{a}}\left(\vec{a} \cdot \vec{\partial}_{\vec{x}} F(\vec{x})\right)=\vec{\partial}_{\vec{a}}\left[\vec{a} \cdot \vec{\partial}_{\vec{x}} G(f(\vec{x}))\right]^{P 40}=$ $\vec{\partial}_{\vec{a}} \underbrace{\left[f(\vec{a}) \cdot \vec{\partial}_{\vec{x}^{\prime}}\right.}_{\text {scalar }} G\left(\vec{x}^{\prime}\right)]_{\vec{x}^{\prime}=f(\vec{x})}=$

$\left[\vec{\partial}_{\vec{a}}\left(f(\vec{a}) \cdot \vec{\partial}_{\vec{x}^{\prime}}\right) G\left(\vec{x}^{\prime}\right)\right]_{\vec{x}^{\prime}=f(\vec{x})} \stackrel{\text { def } 57}{=}$

$\left.\left[\bar{f}\left(\vec{\partial}_{\vec{x}^{\prime}}\right) G\left(\vec{x}^{\prime}\right)\right]\right|_{\vec{x}^{\prime}=f(\vec{x})}=\bar{f}\left(\vec{\partial}_{\vec{x}^{\prime}}\right) G\left(\vec{x}^{\prime}\right)$.

Proposition 64 (second differential) $\vec{\partial}^{2}{ }_{\bar{x}} F(\vec{x})=\vec{\partial}_{\vec{b}} \vec{\partial}_{\vec{a}} F_{\vec{a} \vec{b}}=\left(\vec{\partial}_{\vec{b}} \cdot \vec{\partial}_{\vec{a}}+\vec{\partial}_{\vec{b}} \wedge \vec{\partial}_{\vec{a}}\right) F_{\vec{a} b}$.

\section{Proof 64}

$\vec{\partial}_{\vec{x}} F(\vec{x}) \stackrel{P 54}{=} \vec{\partial}_{\vec{a}}\left(\vec{a} \cdot \vec{\partial}_{\vec{x}} F(\vec{x})\right)^{\text {def } 13} \vec{\partial}_{\vec{a}} F_{\vec{a}}$

$\Rightarrow \vec{\partial}^{2} F(\vec{x})=\vec{\partial}_{\vec{x}}\left(\vec{\partial}_{\vec{x}} F(\vec{x})\right)=\vec{\partial}_{\vec{x}}\left(\vec{\partial}_{\vec{a}} F_{\vec{a}}\right)^{P 54}=$

$\vec{\partial}_{\vec{b}}[\underbrace{\vec{b} \cdot \dot{\vec{\partial}}_{\vec{x}}}_{\text {scalar op. }}\left(\vec{\partial}_{\vec{a}} \dot{F}_{\vec{a}}\right)]=\vec{\partial}_{\vec{b}} \vec{\partial}_{\vec{a}}\left[\left(\vec{b} \cdot \vec{\partial}_{\vec{x}}\right) F_{\vec{a}}\right]^{\text {def } 41}=$

$\vec{\partial}_{\vec{b}} \vec{\partial}_{\vec{a}} F_{\vec{a} \vec{b}} \stackrel{P 18}{=}\left(\vec{\partial}_{\vec{b}} \cdot \vec{\partial}_{\vec{a}}+\vec{\partial}_{\vec{b}} \wedge \vec{\partial}_{\vec{a}}\right) F_{\vec{a} b} \cdot$

Proposition 65 (integrability condition for vector derivative)

$$
\vec{\partial}_{\vec{x}} \wedge \vec{\partial}_{\vec{x}}=0 \Leftrightarrow F_{\vec{a} \vec{b}}=F_{\vec{b} \vec{a}} .
$$

\section{Proof 65}

$(\Rightarrow)$

$\vec{\partial}_{\vec{x}} \wedge \vec{\partial}_{\vec{x}}=0 \Rightarrow 0=(\vec{a} \wedge \vec{b}) \cdot\left(\vec{\partial}_{\vec{x}} \wedge \vec{\partial}_{\vec{x}}\right) F \stackrel{(42)}{=}$ $\vec{a} \cdot\left[\vec{b} \cdot\left(\vec{\partial}_{\vec{x}} \wedge \vec{\partial}_{\vec{x}}\right)\right] F=$

$\vec{a} \cdot\left[\left(\vec{b} \cdot \vec{\partial}_{\vec{x}}\right) \vec{\partial}_{\vec{x}} F-\vec{\partial}_{\vec{x}}\left(\vec{b} \cdot \vec{\partial}_{\vec{x}}\right)\right] F=$

$\left[\left(\vec{b} \cdot \vec{\partial}_{\vec{x}}\right)\left(\vec{a} \cdot \vec{\partial}_{\vec{x}}\right) F-\left(\vec{a} \cdot \vec{\partial}_{\vec{x}}\right)\left(\vec{b} \cdot \vec{\partial}_{\vec{x}}\right)\right] F \stackrel{\text { def } 41}{=}$

$F_{\vec{a} \vec{b}}-F_{\vec{b} \vec{a}}$,

$(\Leftarrow)$ integrability (P. 42): $F_{\vec{a} \vec{b}}=F_{\vec{b} \vec{a}}$

$0=\frac{1}{2}\left(\vec{\partial}_{\vec{b}} \vec{\partial}_{\vec{a}} F_{\vec{a} \vec{b}}-\vec{\partial}_{\vec{a}} \vec{\partial}_{\vec{b}} F_{\vec{b} \vec{a}}\right) \stackrel{P 42}{=}$

$\frac{1}{2}\left(\vec{\partial}_{\vec{b}} \vec{\partial}_{\vec{a}}-\vec{\partial}_{\vec{a}} \vec{\partial}_{\vec{b}}\right) F_{\vec{a} \vec{b}}=\vec{\partial}_{\vec{b}} \wedge \vec{\partial}_{\vec{a}} F_{\vec{a} \vec{b}} \stackrel{P 64}{=}$

$\vec{\partial}_{\vec{x}} \wedge \vec{\partial}_{\vec{x}} F(\vec{x}) \Rightarrow \vec{\partial}_{\vec{x}} \wedge \vec{\partial}_{\vec{x}}=0$.

Proposition 66 (Laplacian) Integrability of $F \Leftrightarrow \vec{\partial}_{\vec{x}}{ }^{2}=\vec{\partial}_{\vec{x}} \cdot \vec{\partial}_{\vec{x}}$.

\section{Proof 66}

Integrability of $F \stackrel{P 65}{\Leftrightarrow} \vec{\partial}_{\vec{x}} \wedge \vec{\partial}_{\vec{x}}=0$

$\Leftrightarrow \vec{\partial}_{\vec{x}}{ }_{(6)}^{(18)} \underset{{ }_{(6)}}{=} \vec{\partial}_{\vec{x}} \cdot \vec{\partial}_{\vec{x}} \wedge \vec{\partial}_{\vec{x}}=\vec{\partial}_{\vec{x}} \cdot \vec{\partial}_{\vec{x}}$.

\section{Proposition 67}

$$
\vec{\partial}_{\vec{x}} \wedge \vec{x}=0 .
$$

\section{Proof 67}

$\vec{\partial}_{\vec{x}} \wedge \vec{x}=\frac{1}{2} \vec{\partial}_{\vec{x}} \wedge(2 \vec{x}) \stackrel{P 53}{=} \frac{1}{2}\left(\vec{\partial}_{\vec{x}} \wedge \vec{\partial}_{\vec{x}}\right) \vec{x}^{2} \stackrel{P 65}{=} 0$, because $F=\vec{x}^{2}$ is integrable.

Proposition 68

$$
\vec{\partial}_{\vec{x}} \vec{x}=\vec{\partial}_{\vec{x}} \cdot \vec{x}=n .
$$

\section{Proof 68}

$\vec{\partial}_{\vec{x}} \vec{x} \stackrel{P 47}{=} \vec{\partial}_{\vec{x}} \cdot \vec{x}+\vec{\partial}_{\vec{x}} \wedge \vec{x}^{P 67} \vec{\partial}_{\vec{x}} \cdot \vec{x}=$ $\left(\sum_{k=1}^{n} \vec{a}^{k} \vec{a}_{k} \cdot \vec{\partial}_{\vec{x}}\right) \cdot \vec{x}=\sum_{k=1}^{n} \vec{a}^{k} \cdot\left(\vec{a}_{k} \cdot \vec{\partial}_{\vec{x}}\right) \vec{x}=$ $\sum_{k=1}^{n} \vec{a}^{k} \cdot P\left(\vec{a}_{k}\right)=\sum_{k=1}^{n} \vec{a}^{k} \cdot \vec{a}_{k}=n$.

Proposition 69

$$
\vec{\partial}_{\vec{x}}|\vec{x}|^{k}=k|\vec{x}|^{k-2} \vec{x} .
$$

\section{Proof 69}

$\vec{\partial}_{\vec{x}}|\vec{x}|^{k} \stackrel{P 55}{=} \hat{x} \frac{d|\vec{x}|^{k}}{d|\vec{x}|}=\hat{x} k|\vec{x}|^{k-1}=k|\vec{x}|^{k-2}|\vec{x}| \hat{x}=k|\vec{x}|^{k-2} \vec{x}$.

\section{Proposition 70}

$$
\vec{\partial}_{\vec{x}}\left(\frac{\vec{x}}{|\vec{x}|^{k}}\right)=\frac{n-k}{|\vec{x}|^{k}} .
$$

\section{Proof 70}




$$
\begin{aligned}
& \vec{\partial}_{\vec{x}}\left(\frac{\vec{x}}{|\vec{x}|^{k}}\right)^{P 52}=\vec{\partial}_{\vec{x}}(\vec{x}) \frac{1}{|\vec{x}|^{k}}+\vec{x} \vec{\partial}_{\vec{x}}|\vec{x}|^{-k}= \\
& n \frac{1}{|\vec{x}|^{k}}+\vec{x}(-k)|\vec{x}|^{-k-2} \vec{x}=n \frac{1}{|\vec{x}|^{k}}-k|\vec{x}|^{-k-2} \vec{x}^{2}= \\
& \frac{n}{|\vec{x}|^{k}}-k|\vec{x}|^{-k}=\frac{n-k}{|\vec{x}|^{k}} .
\end{aligned}
$$

\section{Proposition 71}

$$
\vec{\partial}_{\vec{x}} \log |\vec{x}|=\frac{\vec{x}}{|\vec{x}|^{2}}=\vec{x}^{-1}
$$

\section{Proof 71}

$\vec{\partial}_{\vec{x}} \log |\vec{x}|=\hat{x} \frac{d \log |\vec{x}|}{d|\vec{x}|}=\hat{x} \frac{1}{|\vec{x}|} \stackrel{(12)}{=} \frac{\vec{x}}{|\vec{x}|^{2}} \stackrel{(12)}{=} \vec{x}^{-1}$.

\section{Proposition 72}

For $A=P(A)=\langle A\rangle_{r}$ :

$$
\dot{\vec{\partial}}_{\vec{x}}(\dot{\vec{x}} \cdot A)=A \cdot \vec{\partial}_{\vec{x}} \vec{x}=r A
$$

\section{Proof 72}

If $A$ is a simple r-blade, then

$\dot{\vec{\partial}}_{\vec{x}}(\dot{\vec{x}} \cdot A) \stackrel{(39)}{=} \dot{\vec{\partial}}_{\vec{x}}(A \cdot \dot{\vec{x}})(-1)^{r-1}=$

$\frac{1}{2}[\dot{\vec{\partial}}_{\vec{x}} A \dot{\vec{x}}-(-1)^{r} \underbrace{\left(\vec{\partial}_{\vec{x}} \vec{x}\right)}_{=n(P 68)} A](-1)^{r-1}=$

$\frac{1}{2}\left[\dot{\vec{\partial}}_{\vec{x}} A \dot{\vec{x}}-(-1)^{r} A\left(\vec{\partial}_{\vec{x}} \vec{x}\right)\right](-1)^{r-1}=$

$\left(\dot{\vec{\partial}}_{\vec{x}} \cdot A\right) \dot{\vec{x}}(-1)^{r-1} \stackrel{(39)}{=}\left(A \cdot \vec{\partial}_{\vec{x}}\right) \vec{x}$,

$\stackrel{(30)}{\Rightarrow} A^{-1} \dot{\vec{\partial}}_{\vec{x}}(\dot{\vec{x}} \cdot A)=A^{-1}\left(A \cdot \vec{\partial}_{\vec{x}}\right) \vec{x} \stackrel{(35)}{=} P_{A}\left(\vec{\partial}_{\vec{x}}\right) \vec{x}$

$\stackrel{P 18}{=}\left(\sum_{k=1}^{r} \vec{a}^{k} \vec{a}_{k} \cdot \partial_{\vec{x}}\right) \vec{x}=\sum_{k=1}^{r} \vec{a}^{k}\left(\vec{a}_{k} \cdot \partial_{\vec{x}}\right) \vec{x}=\sum_{k=1}^{P 43} \vec{a}^{k} \vec{a}_{k}$

$\stackrel{\text { (33) }}{=} r \underset{(30)}{A A^{-1}=1} \dot{\vec{\partial}}_{\vec{x}}(\dot{\vec{x}} \cdot A)=\left(A \cdot \vec{\partial}_{\vec{x}}\right) \vec{x}=r A$.

Last step: Multiplication with $A$ from the left. Distributivity (19), (25) gives the same result even for non-simple $A$.

\section{Proposition 73}

For $A=P(A)=\langle A\rangle_{r}$ :

$$
\dot{\vec{\partial}}_{\vec{x}}(\dot{\vec{x}} \wedge A)=A \wedge \vec{\partial}_{\vec{x}} \vec{x}=(n-r) A \text {. }
$$

\section{Proof 73}

If $A$ is a simple r-blade, then

$\dot{\vec{\partial}}_{\vec{x}}(\dot{\vec{x}} \wedge A) \stackrel{(40)}{=} \dot{\vec{\partial}}_{\vec{x}}(A \wedge \dot{\vec{x}})(-1)^{r}=$ $=\frac{1}{2}[\dot{\vec{\partial}} \overrightarrow{\vec{x}} A \dot{\vec{x}}+(-1)^{r} \underbrace{\left(\vec{\partial}_{\vec{x}} \vec{x}\right)}_{=n(P 68)} A](-1)^{r}=$

$\frac{1}{2}\left[\dot{\vec{\partial}}_{\vec{x}} A \dot{\vec{x}}+(-1)^{r} A\left(\vec{\partial}_{\vec{x}} \vec{x}\right)\right](-1)^{r}=$

$\left(\dot{\vec{\partial}}_{\vec{x}} \wedge A\right) \dot{\vec{x}}(-1)^{r} \stackrel{(40)}{=}\left(A \wedge \vec{\partial}_{\vec{x}}\right) \vec{x}$,

$\stackrel{(30)}{\Rightarrow} A^{-1} \dot{\vec{\partial}}_{\vec{x}}(\dot{\vec{x}} \wedge A)=A^{-1}\left(A \wedge \vec{\partial}_{\vec{x}}\right) \vec{x}=$

$A^{-1} I^{-1} I\left(A \wedge \vec{\partial}_{\vec{x}}\right) \vec{X} \stackrel{\text { I pseudo }}{=}$

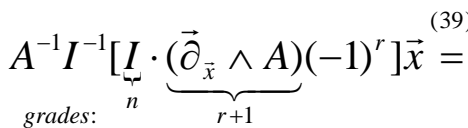

$A^{-1} I^{-1}\left[\left(\vec{\partial}_{\vec{x}} \wedge A\right) \cdot I\right] \vec{x}(-1)^{r+(r+1)(n-r-1)} \stackrel{(38)}{=}$

$=A^{-1} I^{-1}\left[\vec{\partial}_{\vec{x}} \cdot(A I)\right] \vec{x}(-1)^{r+(r+1)(n-r)-r-1} \stackrel{(39)}{=}$

$=A^{-1} I^{-1}[\underbrace{(A I)}_{n-r} \cdot \vec{\partial}_{\vec{x}}] \vec{x}(-1)^{(r+1)(n-r)-1+n-r-1} \stackrel{(40)}{=}$

$=A^{-1} I^{-1}[\underbrace{(I A)}_{n-r} \cdot \vec{\partial}_{\vec{x}}] \vec{x} \underbrace{(-1)^{r(n-r)+r(n-r)}}_{=+1} \stackrel{\text { (35),(36) }}{=}$

$P_{I A}\left(\vec{\partial}_{\vec{x}}\right) \vec{x}^{\text {proof }}=(n-r)$

$\underset{(30)}{\Rightarrow A^{-1}=1} \dot{\vec{\partial}}_{\vec{x}}(\dot{\vec{x}} \wedge A)=\left(A \wedge \vec{\partial}_{\vec{x}}\right) \vec{x}=(n-r) A$.

Last step: Multiplication with $A$ from the left. The distributive rule for the inner product gives the same result even for non-simple $A$.

\section{Proposition 74}

For $A=P(A)=\langle A\rangle_{r}$ :

$$
\dot{\vec{\partial}}_{\vec{x}} A \dot{\vec{x}}=\sum_{k=1}^{n} \vec{a}^{k} A \vec{a}_{k}=(-1)^{r}(n-2 r) A .
$$

\section{Proof 74}

$\dot{\vec{\partial}}_{\vec{x}} A \dot{\vec{x}}=\sum_{k=1}^{P 18} \vec{a}^{k}\left(\vec{a}_{k} \cdot \dot{\vec{\partial}}_{\vec{x}}\right) A \dot{\vec{x}}=\sum_{k=1}^{n} \vec{a}^{k} A\left(\vec{a}_{k} \cdot \dot{\vec{\partial}}_{\vec{x}}\right) \dot{\vec{x}}$

$\stackrel{P 43}{=} \sum_{k=1}^{n} \vec{a}^{k} A \vec{a}_{k}$

$\dot{\vec{\partial}}_{\vec{x}} A \dot{\vec{x}}=\dot{\vec{\partial}}_{\vec{x}}[A \cdot \dot{\vec{x}}+A \wedge \dot{\vec{x}}]^{(39),(40)}=$

$\dot{\vec{\partial}}_{\vec{x}}\left[\dot{\vec{x}} \cdot A(-1)^{r-1}+\dot{\vec{x}} \wedge A(-1)^{r}\right]=$

$(-1)^{r}\left[-\dot{\vec{\partial}}_{\vec{x}}(\dot{\vec{x}} \cdot A)+\dot{\vec{\partial}}_{\vec{x}}(\dot{\vec{x}} \wedge A)\right] \underset{P 73}{=}$

$(-1)^{r}[-r A+(n-r) A]=(-1)^{r}(n-2 r) A$. 


\section{Proposition 75}

For $\vec{a}=\vec{a}(\vec{x}), \vec{b}=\vec{b}(\vec{x})$ :

$\vec{\partial}_{\vec{x}}(\vec{a} \cdot \vec{b})$

$=\vec{a} \cdot \vec{\partial}_{\vec{x}} \vec{b}+\vec{b} \cdot \vec{\partial}_{\vec{x}} \vec{a}-\vec{a} \cdot\left(\vec{\partial}_{\vec{x}} \wedge \vec{b}\right)-\vec{b} \cdot\left(\vec{\partial}_{\vec{x}} \wedge \vec{a}\right)$.

Proof 75

$\vec{a} \cdot\left(\vec{\partial}_{\vec{x}} \wedge \vec{b}\right) \underset{(41)}{=} \vec{a} \cdot \dot{\vec{\partial}}_{\vec{x}} \dot{\vec{b}}-\dot{\vec{\partial}}_{\vec{x}}(\dot{\vec{b}} \cdot \vec{a})$,

$\vec{b} \cdot\left(\vec{\partial}_{\vec{x}} \wedge \vec{a}\right) \underset{(41)}{=P 18} \vec{b} \cdot \dot{\vec{\partial}}_{\vec{x}} \dot{\vec{a}}-\dot{\vec{\partial}}_{\vec{x}}(\dot{\vec{a}} \cdot \vec{b})$,

$\stackrel{\text { addition }}{\Rightarrow} \vec{\partial}_{\vec{x}}(\vec{a} \cdot \vec{b})=\dot{\vec{\partial}}_{\vec{x}}(\dot{\vec{a}} \cdot \vec{b})+\dot{\vec{\partial}}_{\vec{x}}(\dot{\vec{b}} \cdot \vec{a})=$ $\vec{a} \cdot \vec{\partial}_{\vec{x}} \vec{b}+\vec{b} \cdot \vec{\partial}_{\vec{x}} \vec{a}-\vec{a} \cdot\left(\vec{\partial}_{\vec{x}} \wedge \vec{b}\right)-\vec{b} \cdot\left(\vec{\partial}_{\vec{x}} \wedge \vec{a}\right)$.

Definition 76 (Lie bracket)

For $\vec{a}=\vec{a}(\vec{x}), \vec{b}=\vec{b}(\vec{x})$ :

$$
[\vec{a}, \vec{b}] \equiv \vec{a} \cdot \vec{\partial}_{\vec{x}} \vec{b}-\vec{b} \cdot \vec{\partial}_{\vec{x}} \vec{a} .
$$

\section{Proposition 77}

$$
[\vec{a}, \vec{b}]=\vec{\partial}_{\vec{x}} \cdot(\vec{a} \wedge \vec{b})-\vec{b} \vec{\partial}_{\vec{x}} \cdot \vec{a}+\vec{a} \vec{\partial}_{\vec{x}} \cdot \vec{b} .
$$

\section{Proof 77}

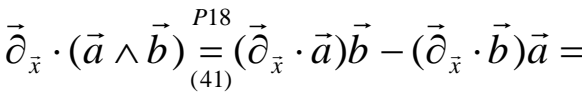

$\vec{b}\left(\vec{\partial}_{\vec{x}} \cdot \vec{a}\right)+\left(\vec{a} \cdot \vec{\partial}_{\vec{x}}\right) \vec{b}-\vec{a}\left(\vec{\partial}_{\vec{x}} \cdot \vec{b}\right)-\left(\vec{b} \cdot \vec{\partial}_{\vec{x}}\right) \vec{a}$

$\Rightarrow[\vec{a}, \vec{b}]=\left(\vec{a} \cdot \vec{\partial}_{\vec{x}}\right) \vec{b}-\left(\vec{b} \cdot \vec{\partial}_{\vec{x}}\right) \vec{a}=$

$\vec{\partial}_{\vec{x}} \cdot(\vec{a} \wedge \vec{b})-\vec{b} \vec{\partial}_{\vec{x}} \cdot \vec{a}+\vec{a} \vec{\partial}_{\vec{x}} \cdot \vec{b}$.

\section{Proposition 78}

For $\vec{a}=\vec{a}(\vec{x}), \vec{b}=\vec{b}(\vec{x}), \vec{c}=\vec{c}(\vec{x})$ :

$(\vec{c} \wedge \vec{b}) \cdot\left(\vec{\partial}_{\vec{x}} \wedge \vec{a}\right)=\vec{b} \cdot \dot{\vec{\partial}}_{\vec{x}} \dot{\vec{a}} \cdot \vec{c}-\vec{c} \cdot \dot{\vec{\partial}}_{\vec{x}} \dot{\vec{a}} \cdot \vec{b}$

$=\vec{b} \cdot \vec{\partial}_{\vec{x}}(\vec{a} \cdot \vec{c})-\vec{c} \cdot \vec{\partial}_{\vec{x}}(\vec{a} \cdot \vec{b})+[\vec{c}, \vec{b}] \cdot \vec{a}$.

\section{Proof 78}

$(\vec{c} \wedge \vec{b}) \cdot\left(\vec{\partial}_{\vec{x}} \wedge \vec{a}\right) \stackrel{(42)}{=} \vec{c} \cdot\left(\vec{b} \cdot\left(\vec{\partial}_{\vec{x}} \wedge \vec{a}\right)\right)^{\text {proof } 75}=$

$\vec{c} \cdot\left(\vec{b} \cdot \dot{\vec{\partial}}_{\vec{x}} \dot{\vec{a}}-\dot{\vec{\partial}}_{\vec{x}}(\dot{\vec{a}} \cdot \vec{b})\right)=\vec{b} \cdot \dot{\vec{\partial}}_{\vec{x}} \dot{\vec{a}} \cdot \vec{c}-\vec{c} \cdot \dot{\vec{\partial}}_{\vec{x}} \dot{\vec{a}} \cdot \vec{b}$

$=\vec{b} \cdot \vec{\partial}_{\vec{x}}(\vec{a} \cdot \vec{c})-\vec{b} \cdot \dot{\vec{\partial}}_{\vec{x}}(\vec{a} \cdot \dot{\vec{c}})$

$-\vec{c} \cdot \vec{\partial}_{\vec{x}}(\vec{a} \cdot \vec{b})+\vec{c} \cdot \dot{\vec{\partial}}_{\vec{x}}(\vec{a} \cdot \dot{\vec{b}})=$

$\vec{b} \cdot \vec{\partial}_{\vec{x}}(\vec{a} \cdot \vec{c})-\vec{c} \cdot \vec{\partial}_{\vec{x}}(\vec{a} \cdot \vec{b})$

$+\vec{c} \cdot \dot{\vec{\partial}}_{\vec{x}}(\dot{\vec{b}} \cdot \vec{a})-\vec{b} \cdot \dot{\vec{\partial}}_{\vec{x}}(\dot{\vec{c}} \cdot \vec{a}) \stackrel{\text { def } 76}{=}$ $=\vec{b} \cdot \vec{\partial}_{\vec{x}}(\vec{a} \cdot \vec{c})-\vec{c} \cdot \vec{\partial}_{\vec{x}}(\vec{a} \cdot \vec{b})+[\vec{c}, \vec{b}] \cdot \vec{a}$.

\section{Proposition 79}

For $\vec{a}=\vec{a}(\vec{x}), \vec{b}=\vec{b}(\vec{x})$ :

$$
\vec{a} \cdot\left(\vec{\partial}_{\vec{x}} \wedge \vec{b}\right)=\dot{\vec{b}} \cdot\left(\dot{\vec{\partial}}_{\vec{x}} \wedge \vec{a}\right)+\dot{\vec{\partial}}_{\vec{x}} \cdot(\vec{a} \wedge \dot{\vec{b}})=
$$$$
\left(\vec{a} \wedge \vec{\partial}_{\vec{x}}\right) \cdot \vec{b}+\vec{a} \cdot \vec{\partial}_{\vec{x}} \vec{b}-\vec{a} \vec{\partial}_{\vec{x}} \cdot \vec{b} .
$$

\section{Proof 79}

Jacobi identity (44) $\Rightarrow$

$\vec{a} \cdot\left(\vec{\partial}_{\vec{x}} \wedge \vec{b}\right)+\dot{\vec{\partial}}_{\vec{x}} \cdot(\dot{\vec{b}} \wedge \vec{a})+\dot{\vec{b}} \cdot\left(\vec{a} \wedge \dot{\vec{\partial}}_{\vec{x}}\right)=0 \Rightarrow$

$\vec{a} \cdot\left(\vec{\partial}_{\vec{x}} \wedge \vec{b}\right)=\dot{\vec{b}} \cdot\left(\dot{\vec{\partial}}_{\vec{x}} \wedge \vec{a}\right)+\dot{\vec{\partial}}_{\vec{x}} \cdot(\vec{a} \wedge \dot{\vec{b}}) \underset{(39)}{\left({ }^{(41)}\right.}$

$\left(\vec{a} \wedge \vec{\partial}_{\vec{x}}\right) \cdot \vec{b}+\vec{a} \cdot \vec{\partial}_{\vec{x}} \vec{b}-\vec{a} \vec{\partial}_{\vec{x}} \cdot \vec{b}$.

\section{Proposition 80}

For $\vec{a}=\vec{a}(\vec{x}), \vec{b}=\vec{b}(\vec{x})$ :

$\dot{\vec{\partial}}_{\vec{x}} \cdot(\vec{a} \wedge \dot{\vec{b}})=\dot{\vec{a}} \cdot\left(\dot{\vec{\partial}}_{\vec{x}} \wedge \dot{\vec{b}}\right)-\dot{\vec{b}} \cdot\left(\dot{\vec{\partial}}_{\vec{x}} \wedge \dot{\vec{a}}\right)=$

$$
\left(\vec{b} \wedge \vec{\partial}_{\vec{x}}\right) \cdot \vec{a}+\vec{a} \cdot\left(\vec{\partial}_{\vec{x}} \wedge \vec{b}\right)
$$$$
-\left(\vec{a} \wedge \vec{\partial}_{\vec{x}}\right) \cdot \vec{b}-\vec{b} \cdot\left(\vec{\partial}_{\vec{x}} \wedge \vec{a}\right) .
$$

\section{Proof 80}

Jacobi identity (44) $\Rightarrow$

$\dot{\vec{\partial}}_{\vec{x}} \cdot(\vec{a} \wedge \dot{\vec{b}})+\dot{\vec{a}} \cdot\left(\dot{\vec{b}} \wedge \dot{\vec{\partial}}_{\vec{x}}\right)+\dot{\vec{b}} \cdot\left(\dot{\vec{\partial}}_{\vec{x}} \wedge \dot{\vec{a}}\right)=0 \Rightarrow$

$\dot{\vec{\partial}}_{\vec{x}} \cdot(\vec{a} \wedge \dot{\vec{b}})=\dot{\vec{a}} \cdot\left(\dot{\vec{\partial}}_{\vec{x}} \wedge \dot{\vec{b}}\right)-\dot{\vec{b}} \cdot\left(\dot{\vec{\partial}}_{\vec{x}} \wedge \dot{\vec{a}}\right)=$

$\dot{\vec{a}} \cdot\left(\dot{\vec{\partial}}_{\vec{x}} \wedge \vec{b}\right)+\vec{a} \cdot\left(\dot{\vec{\partial}}_{\vec{x}} \wedge \dot{\vec{b}}\right)$

$-\dot{\vec{b}} \cdot\left(\dot{\vec{\partial}}_{\vec{x}} \wedge \vec{a}\right)-\vec{b} \cdot\left(\dot{\vec{\partial}}_{\vec{x}} \wedge \dot{\vec{a}}\right)=$

$\left(\vec{b} \wedge \vec{\partial}_{\vec{x}}\right) \cdot \vec{a}+\vec{a} \cdot\left(\vec{\partial}_{\vec{x}} \wedge \vec{b}\right)$

$-\left(\vec{a} \wedge \vec{\partial}_{\vec{x}}\right) \cdot \vec{b}-\vec{b} \cdot\left(\vec{\partial}_{\vec{x}} \wedge \vec{a}\right)$.

\section{Proposition 81}

$A \times\left(\vec{\partial}_{\vec{x}} \wedge \vec{b}\right)=$

$A \cdot \vec{\partial}_{\vec{x}} \vec{b}-\dot{\vec{\partial}}_{\vec{x}} \dot{\vec{b}} \cdot A=A \wedge \vec{\partial}_{\vec{x}} \vec{b}-\dot{\vec{\partial}}_{\vec{x}} \dot{\vec{b}} \wedge A$.

with the commutator product $A \times B$ of multivectors $A, B$ (45).

\section{Proof 81}

$A \times\left(\vec{\partial}_{\vec{x}} \wedge \vec{b}\right) \stackrel{(45)}{=}-\left(\dot{\vec{\partial}}_{\vec{x}} \wedge \dot{\vec{b}}\right) \times A^{(46)}=$

$-\dot{\vec{\partial}}_{\vec{x}} \dot{\vec{b}} \cdot A+A \cdot \vec{\partial}_{\vec{x}} \vec{b}^{(46)}=-\dot{\vec{\partial}}_{\vec{x}} \dot{\vec{b}} \wedge A+A \wedge \vec{\partial}_{\vec{x}} \vec{b}$.

\section{Proposition 82}

For $A=\langle A\rangle_{r}=A(\vec{x}), \quad B=\langle B\rangle_{s}=B(\vec{x})$ : 


$$
\begin{aligned}
& \dot{A} \wedge \dot{\vec{\partial}}_{\vec{x}} \wedge \dot{B}=(-1)^{r} \vec{\partial}_{\vec{x}} \wedge(A \wedge B)= \\
& A \wedge \vec{\partial}_{\vec{x}} \wedge B+(-1)^{r+s(r+1)} B \wedge \vec{\partial}_{\vec{x}} \wedge A .
\end{aligned}
$$

\section{Proof 82}

$\dot{A} \wedge \dot{\vec{\partial}}_{\vec{x}} \wedge \dot{B}^{(40)}=(-1)^{r} \vec{\partial}_{\vec{x}} \wedge(A \wedge B)$,

$\dot{A} \wedge \dot{\vec{\partial}}_{\vec{x}} \wedge \dot{B}=A \wedge \vec{\partial}_{\vec{x}} \wedge B+\left(\dot{A} \wedge \dot{\vec{\partial}}_{\vec{x}}\right) \wedge B=$

$A \wedge \vec{\partial}_{\vec{x}} \wedge B+(-1)^{r}\left(\dot{\vec{\partial}}_{\vec{x}} \wedge \dot{A}\right) \wedge B \stackrel{(40)}{=}$

$A \wedge \vec{\partial}_{\vec{x}} \wedge B+(-1)^{r+s(r+1)} B \wedge\left(\vec{\partial}_{\vec{x}} \wedge A\right)$.

\section{Conclusion}

This article first summarized important geometric algebra relationships, which are necessary for the thorough and explicit development of the vector differential calculus part of universal geometric calculus.

It then showed how to differentiate multivector functions by a vector, including the results of standard vector analysis. The vector differential relationships are proven in a very explicit step by step way, enabling the reader, who is unfamiliar with the algebraic techniques to get complete comprehension. It may thus serve as important reference material for studying and applying vector differential calculus.

Future work in a similar manner should be done to elucidate the calculus with multivector derivatives.

\section{Acknowledgements}

"Soli deo gloria."

(Latin: To God alone be the glory.) [8]

I thank my wife for her patient support and K. Shinoda (Kyoto), H. Shitaka (Hiroshima) and H. Honma (Fukui) for their prayer support.

\section{References}

[1] D. Hestenes, New Foundations for Classical Mechanics, $2^{\text {nd }}$ ed., Kluwer Academic Publishers, Dordrecht, 1999.

[2] D. Hestenes, G. Sobczyk, Clifford Algebra to Geometric Calculus, Kluwer Academic Publishers, Dordrecht, 1999 reprint.

[3] E. Hitzer, Antisymmetric Matrices Are Real Bivectors, Mem. Fac. Eng. Fukui Univ. 499(2), 283 (2001). http://sinai.mech.fukui-u.ac.jp/gcj/pubs.html

[4] L. Dorst, The inner products of geometric algebra, in C. Doran, L. Dorst and J. Lasenby eds. Applied Geometrical Alegbras in computer Science and Engineering, AGACSE
2001, Birkhauser, 2001.

[5] C. Doran, Geometric Algebra and its Application to Mathematical Physics, Ph.D. thesis, University of Cambridge, 1994.

[6] H. Grassmann, (F. Engel, editor) Die Ausdehnungslehre von 1844 und die Geometrische Analyse, 1, part 1, Teubner, Leipzig, 1894.

[7] Hebrews, chapter 11, verses 1 to 3, Holy Bible, New International Version.

[8] Johann Sebastian Bach. 